\title{
Diffraction effects on diffusive bistable optical arrays and optical memory ${ }^{\text {मे }}$
}

\author{
Yuchi Chen, David W. McLaughlin* \\ Courant Institute of Mathematical Sciences, New York University, 251 Mercer Street, New York, NY 10012, USA
}

Received 18 September 1998; received in revised form 7 June 1999; accepted 30 August 1999

Communicated by C.K.R.T. Jones

\begin{abstract}
Bistable responses of Fabry-Pérot cavities and all-optical arrays, in the presence of weak diffraction and strong diffusion, are studied both analytically and numerically. The model is a pair of nonlinear Schrödinger equations coupled through a diffusion equation. The numerical computations are based on a split-step method with three distinct characteristics. This bistable nonlinear system, with strong diffusion and weak diffraction, behaves very differently than dispersive bistability with Kerr nonlinearity. Nevertheless, focusing nonlinearity can improve its response characteristics significantly. For example, it is found that hysteresis loops are much wider when nonlinearity is self-focusing than when nonlinearity is self-defocusing. For self-defocusing nonlinearity, strong diffraction can close a hysteresis loop completely. Because of strong diffusion, refractive index distributions are smoothed versions of intensity distributions. This weakens the nonlinear behavior of the system. By approximating the refractive index by a constant, the model is reduced to a discrete map. This reduction incorporates diffraction into a nonlinear response function, allowing diffractive effects to be studied analytically, and shown to agree well with more extensive numerical simulations. Optical arrays are also studied numerically. Because of weaker diffractive crosstalk and a wider "operation gap" between "on" bits and "off" bits, an array with focusing nonlinearity allows finer packing than with self-defocusing nonlinearity. Using the reduced map, optimal packing densities of optical arrays are estimated. (C2000 Elsevier Science B.V. All rights reserved.
\end{abstract}

Keywords: Bistable optical array; Diffraction effect

\section{Introduction}

All-optical arrays as potential memory devices [19] have intrigued physicists since the first observation of optical bistability [17]. (For background material see, for example, [1,9,16].) Optical arrays were analyzed theoretically by Firth $[4,5,18]$ in the late 1980 s, using a purely diffusive one-dimensional model for a nonlinear phase shift $\varphi[6,8]$ :

$$
\tau \frac{\partial \varphi}{\partial t}=-\varphi+\delta+\frac{\partial^{2}}{\partial x^{2}} \varphi+2\left(1+r_{b}^{2}\right) f(\varphi) I_{\text {in }}
$$

\footnotetext{
许 Funded in part by AFOSR-90-0161 and by NSF DMS 8922717 A01.

* Corresponding author.

E-mail address:dmac@cims.nyu.edu (D.W. McLaughlin).
} 


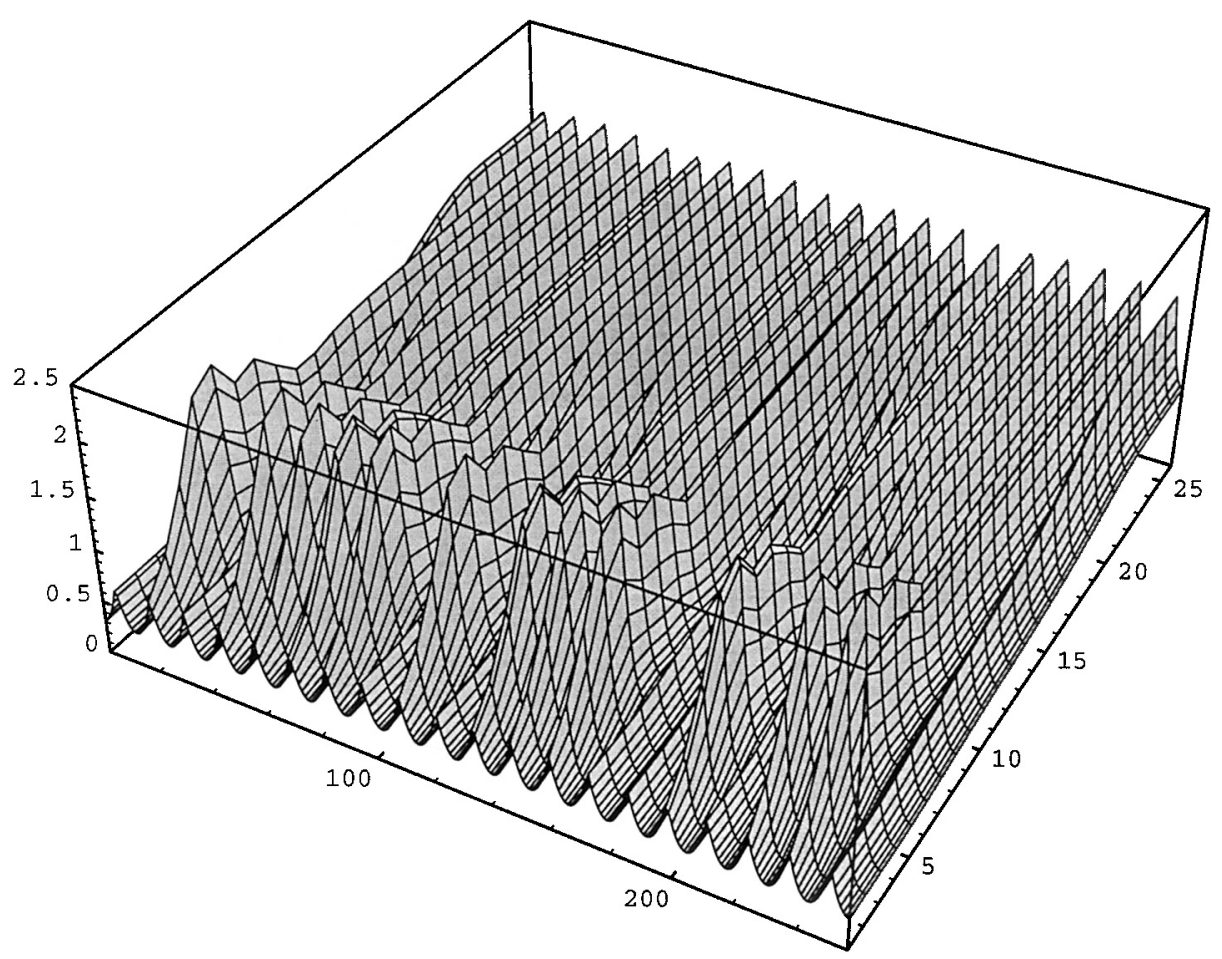

Fig. 1. A defocusing array of 20 pixels with pixel separation $S=3$ is not able to address pattern "01011110101110010110".

Steady state solutions satisfy a non-autonomous Hamiltonian system with the spatial variable $x$ playing the role of time. Such a system could exhibit chaotic behavior. Firth pointed out that this spatial chaos might be advantageous for effective optical memory because it ensured that the system possess a great many states which could be used to store information. That idea established an interesting new connection between chaotic dynamical systems and the area of all-optical memories.

By approximating the input distributions with $\delta$-function spatial profiles located at pixel positions, Firth derived a two-dimensional map which was equivalent to the Hamiltonian system. With this map, mathematical tools in the theory of dynamical systems could be used to explain many properties of optical arrays. In particular, the existence of a Smale horseshoe for the map provided a criterion for effective array operation.

However, realistic models of optical arrays should take into account the effects of finite beam width. Here we are interested in diffractive effects on diffusion dominated media and are focusing upon propagation. In this setting, significant differences in performance occur between focusing and defocusing nonlinearities. Such differences are clearly demonstrated by the simulations shown in Figs. 1-3, where an array of 20 pixels with defocusing nonlinearity is not able to address to the target pattern; while the same array with focusing nonlinearity is addressed successfully with sufficient stability to allow the bit pattern to be reversed.

In this paper, we analyze the effects of diffraction on models of diffusive optical arrays using both numerical and analytical methods. Our results emphasize differences in performance between focusing and defocusing arrays. With fixed input, strong diffraction can destroy bistability for both types of nonlinearity. However, in the focusing case, bistability can be easily re-established by pumping more energy into the system. We also emphasize that our numerical results display a type of nonlinear behavior which is quite distinct from well-known behavior for solutions of the nonliner Schrödinger equation. 


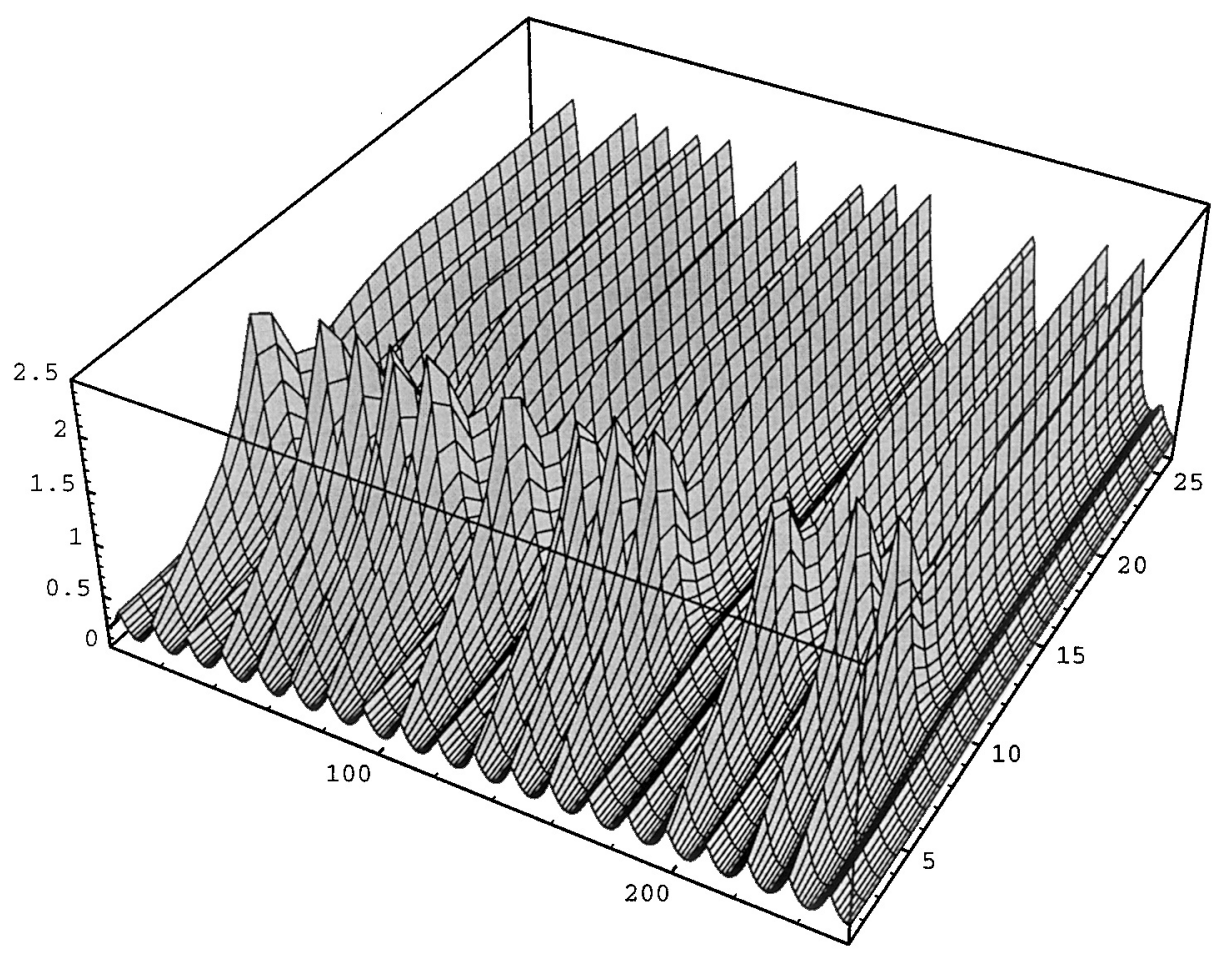

Fig. 2. The same array as Fig. 1 with focusing nonlinearity can address pattern "01011110101110010110".

In order to describe the behavior of the array analytically, in the presence of weak diffraction, we derive a "reduced map" which depends explicitly upon the Fresnel number. (This analytical work can be viewed as an extension of Firth's diffusive model to include weak diffraction.) This reduced map is very useful for understanding the performance of the array. For example, analysis with this map confirms the numerical results - namely, that bistability can survive much stronger diffraction with focusing nonlinearity than with defocusing.

Even though our simulations are restricted to relatively small arrays, they provide some interesting and useful results about optimal packing densities, which can be estimated with this reduced map. Focusing arrays perform better than defocusing, in that they allow higher packing densities - a difference which is even more striking, the wider is the transverse input profile.

Here is an outline for the rest of this paper. Background information is briefly described in Section 2. In Section 3 , we discuss our numerical method and its validation. The details of the algorithm can be found in Appendix A. A comparative study of diffractive effects on optical bistabilities for different types of nonlinearity is given in Section 4. Section 4.4 discusses why strong nonlinear behavior is not observed, as well as differences between the observed behavior and that of the nonlinear Schrödinger equation which is far more familiar. Sections 5.1 and 5.2 reduces the full model to a two-dimensional map, and uses this map to address properties of the system in Sections 5.3 and 5.4. Section 6 describes some numerical and theoretical results on optical arrays. A brief description of some of the results described in this paper may be found in [3].

\subsection{Mathematical model}

Our mathematical model consists of a pair of nonlinear Schrödinger equations for the left and right propagating field envelopes (Fig. 4) coupled through a diffusion equation for the refractive index [2]: 


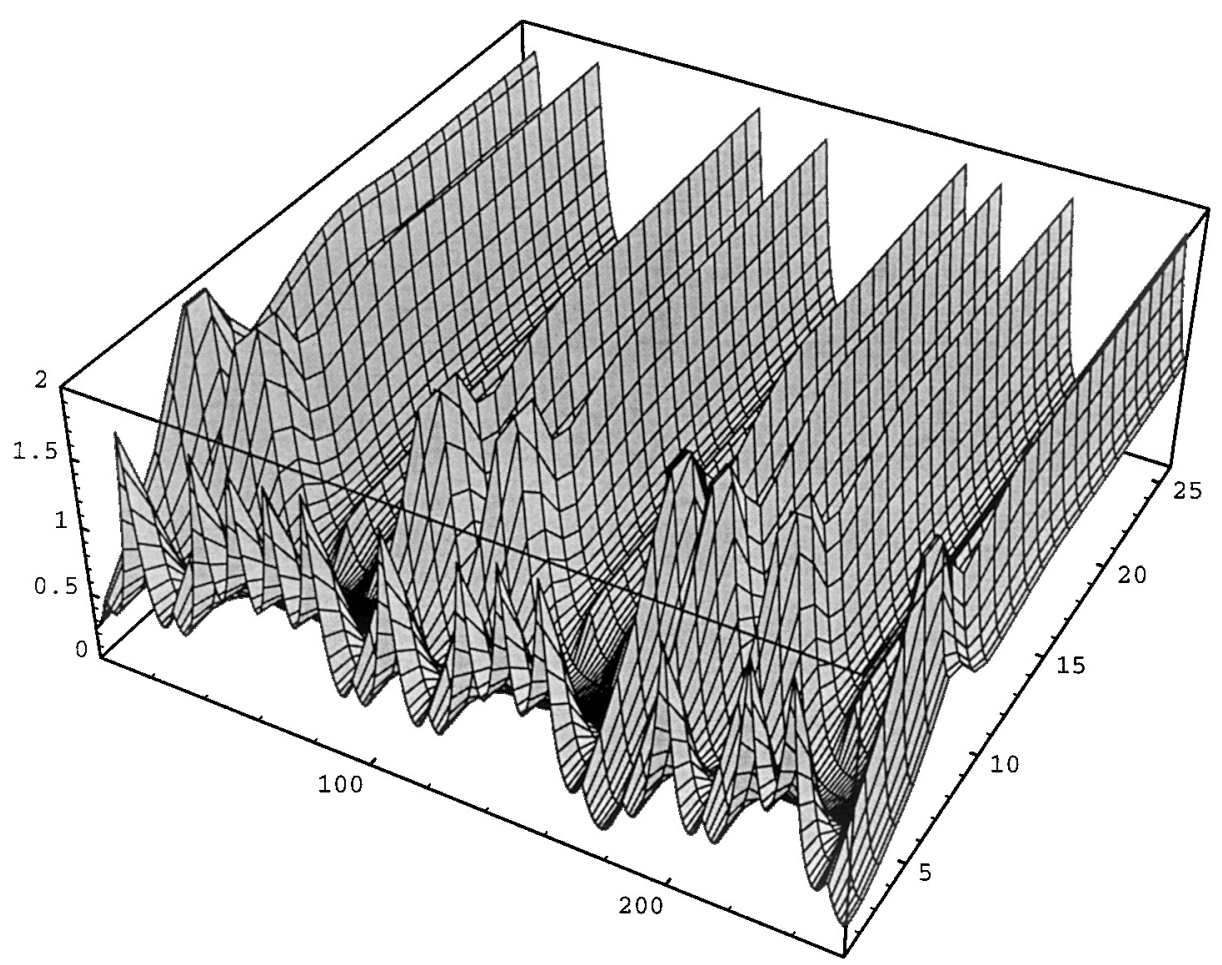

Fig. 3. Bit reversal of Fig. 2.

$$
\begin{aligned}
& \frac{\partial F}{\partial t}+\frac{\partial F}{\partial z}-\mathrm{i} \Gamma \frac{\partial^{2}}{\partial x^{2}} F=\mathrm{i} h F, \\
& \frac{\partial B}{\partial t}-\frac{\partial B}{\partial z}-\mathrm{i} \Gamma \frac{\partial^{2}}{\partial x^{2}} B=\mathrm{i} h B, \\
& \tau \frac{\partial h}{\partial t}-\frac{\partial^{2}}{\partial x^{2}} h+h=n_{2}\left(|F|^{2}+|B|^{2}\right),
\end{aligned}
$$

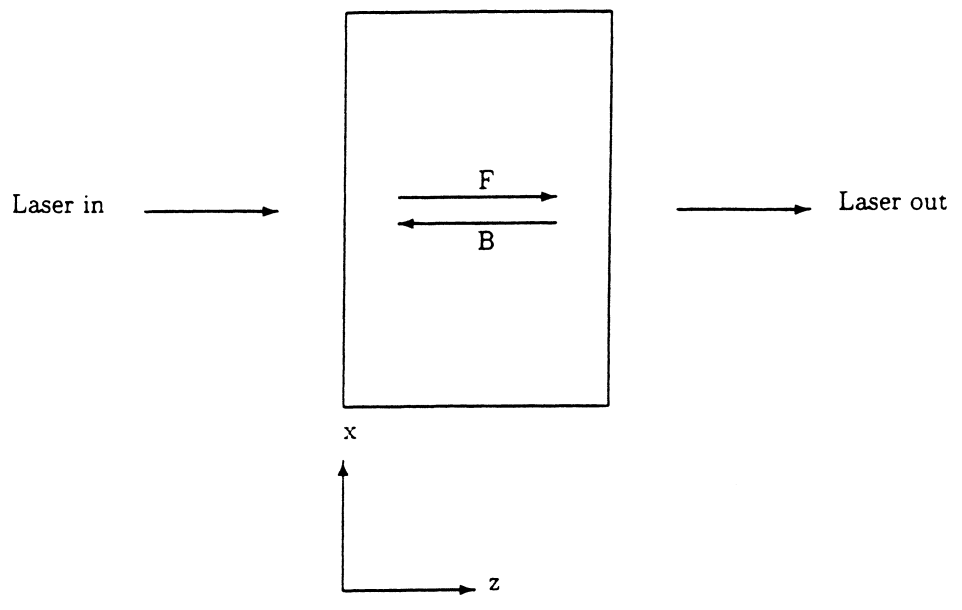

Fig. 4. A simple illustration of a Fabry-Pérot cavity. 
with cavity boundary conditions:

$$
F=r_{\mathrm{f}} B+F_{\text {in }}, \quad z=0, \quad B=r_{\mathrm{b}} \mathrm{e}^{\mathrm{i} \delta} F, \quad z=1 .
$$

Here the longitudinal coordinate is normalized by the depth $L$ of the cavity $(z=Z / L)$, and $\delta=2 k L$ is the linear detuning. Because we are interested in diffusion dominated media, we normalize the transverse coordinate with the diffusion length $l_{\mathrm{D}}\left(x=X / l_{\mathrm{D}}\right)$. Note this yields a coefficient of diffraction $\Gamma\left(=L / 2 k_{0} l_{\mathrm{D}}^{2}\right)$ which is not the usual Fresnel number $\mathcal{F}$. However, $\Gamma$ is related to $\mathcal{F}$ by $\mathcal{F}=\left(2 l_{\mathrm{D}}^{2} / w^{2}\right) \Gamma$, where $w$ is a parameter that measures the width of the input laser pulses.

The normalized carrier density $h$ is related to the nonlinear refractive index by $h=\left(\omega_{0} L / c\right) n^{\mathrm{NL}}$. We assume a medium in which charge carriers are mobile enough that $n^{\mathrm{NL}}$ satisfies a diffusion process driven by electric fields. The field envelopes $F$ and $B$ are normalized so that $n_{2}= \pm 1$ in (1.3), with positive sign for focusing nonlinearity and negative sign for defocusing nonlinearity.

\section{Background information}

\subsection{Plane wave optical bistability}

For a stationary plane wave input, system (1.1)-(1.3) can be reduced to an equation for a nonlinear phase shift $\varphi=\delta+2 h$ :

$$
\varphi-\delta=2\left(1+r_{\mathrm{b}}^{2}\right) f(\varphi) I_{\text {in }},
$$

where $r=r_{\mathrm{f}} r_{\mathrm{b}}, I_{\text {in }}=\left|F_{\text {in }}\right|^{2}$, and

$$
f(\varphi)=\frac{1}{1+r^{2}-2 r \cos (\varphi)}
$$

is the nonlinear response function of the cavity.

Eq. (2.1) is an implicit equation for $\varphi$ which can be solved graphically (see Fig. 5) as the intersections of two curves:

$$
g_{1}(\varphi)=\varphi-\delta, \quad g_{2}(\varphi)=2\left(1+r_{\mathrm{b}}^{2}\right) f(\varphi) I_{\mathrm{in}} .
$$

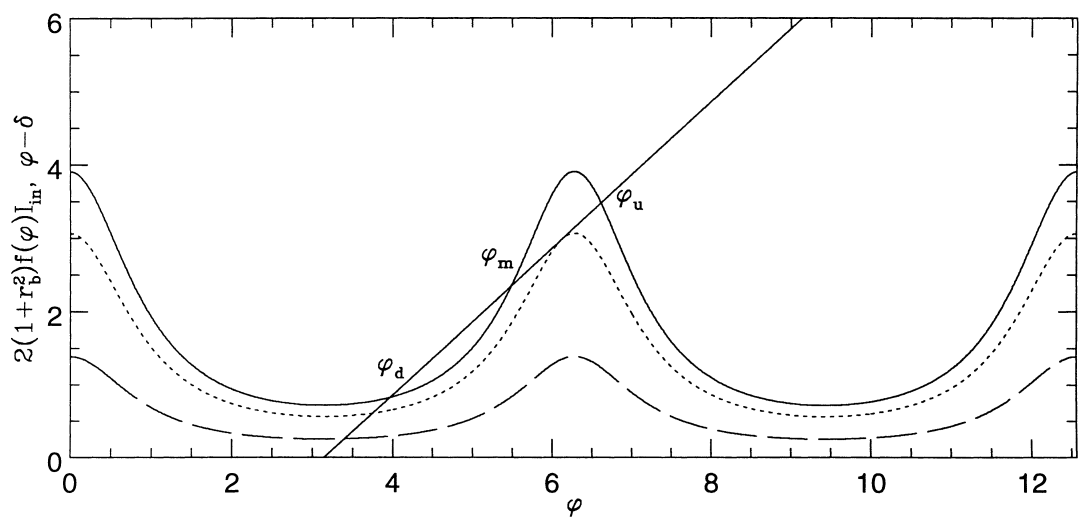

Fig. 5. Bistable states of a nonlinear Fabry-Pérot cavity with plane wave input. $\delta=\pi, r_{\mathrm{f}}=0.8, r_{\mathrm{b}}=0.5, r=0.4$. The solid, dotted and dashed lines are for $I_{\text {in }}=0.5625,0.4415$ and 0.2 , respectively. 


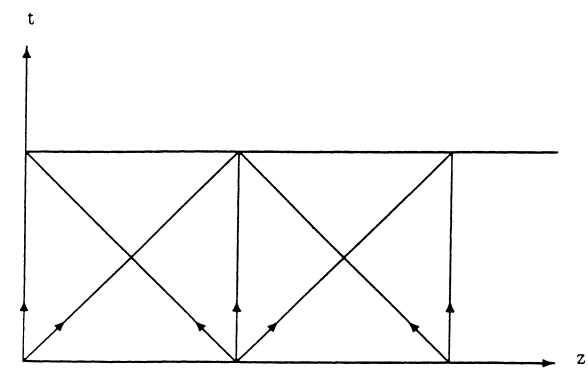

Fig. 6. The characteristic directions of (1.1)-(1.3).

Bistability occurs when the intersection is not unique. Fig. 5 illustrates that, when $\delta=\pi$, there are three intersections: $\varphi_{\mathrm{d}}=3.96744, \varphi_{\mathrm{m}}=5.48977$ and $\varphi_{\mathrm{u}}=6.61824$. At $\varphi_{\mathrm{u}}, B$ is mostly in phase with $F_{\text {in }}$ so the output is strong. While at $\varphi_{\mathrm{d}}, B$ is out of phase with $F_{\text {in }}$ and results in a relatively weaker output. $\varphi_{\mathrm{m}}$ is usually dynamic unstable [2]. Notice that $\delta$ near $\pi$ offers the best chance for the existence of bistability at low input. Hence, $\delta=\pi$ will be used throughout this paper.

\subsection{Diffusion-only model}

An intuitive description of array operation can be given through a diffusion-only model, which can be derived from (1.1)-(1.3) by simply dropping the diffraction terms. In the steady state, the model reduces to a scalar ordinary differential equation for the carrier density:

$$
\frac{\mathrm{d}^{2}}{\mathrm{~d} x^{2}} h(x)-h(x)+\left(1+r_{\mathrm{b}}^{2}\right) f(\delta+2 h(x)) I_{\text {in }}(x)=0 .
$$

Here the transverse coordinate $x$ labels the location of pixels. We assume that the pixels are evenly distributed and are centered at $x_{n}=n S$, where $S$ stands for pixel separation. For a practical optical array, $n$ takes numbers in a finite range. For the moment, we ignore this size limit and assume $n=\ldots,-2,-1,0,1,2, \ldots$

Eq. (2.2) can be viewed as a non-autonomous Hamiltonian system. Such systems are well known for their chaotic behavior. Firth [4,5] pointed out that chaotic behavior in space could be important for array operation, because it ensures that the array possesses a great many bound states with which one can store information. Firth used mathematical tools from the theory of dynamical systems to study optical arrays. For example, he reduced Eq. (2.2) to a two-dimensional mapping:

$$
A_{n+1}=2 c A_{n}-s\left(1+r_{\mathrm{b}}^{2}\right) f\left(\delta+2 A_{n}\right) P_{0}-B_{n}, \quad B_{n+1}=A_{n}
$$

with which maximum array density could be estimated by the existence of a horseshoe. For the same cavity parameters as Fig. 5 and input power $P_{0}=1.125$, the minimum pixel spacing is estimated to be $S_{\mathrm{c}}=2.9340$. In reality, for materials like InSb with $l_{\mathrm{D}}=60 \mu \mathrm{m}, S_{\mathrm{c}}$ would translate into a separation of roughly $180 \mu \mathrm{m}$, or about 55 pixels per centimeter.

\section{Numerical methods}

Our numerical method is based on a "split-step" procedure [10], splitting each equation in (1.1)-(1.3) along its own characteristic directions in the $(t, z)$ plane (Fig. 6). The split-step method is particularly efficient for our system 
because it not only separates nonlinearity from diffraction and diffusion, but it also decouples variables and spatial dimensions. The method is easily parallelized.

The specific algorithm can be found in Appendix A. For validation, we used several explicit solutions and a "diffusion only" approximation for narrow input. For details about these validations, see [2]. A typical run with 20 longitudinal grid points and 256 transverse grid points for 25 roundtrips of propagation requires $20 \mathrm{~min}$ of computer time on a Silicon Graphics' Iris workstation. Some of the larger studies are run on a 16 processor C-90 Cray supercomputer at Pittsburgh Supercomputing Center. For the same typical run, the C-90 is 60 times faster than the Iris workstation.

\section{Bistability}

In reality, no beam is a plane wave and transverse effects must be taken into account. These produce a subtle combination of refraction, diffraction and diffusion - all of which require numerical investigation. In a diffraction-only system, plane wave solutions were found to be good approximations before switching up [6]. However, when the center intensity of the beam reaches the switching-up threshold of plane waves, diffraction will generate a switching wave which propagates outwards [6]. This wave stops when that portion of the beam for which an upper branch exists is switched up. After the switching wave stops, plane wave approximations can again apply to both the central plateau of the beam and the tail. Thus, for diffraction-only systems, the plane wave approximations fails only in the transition region.

In this section, we will study bistability with both diffraction and diffusion present, as described mathematically by Eqs. (1.1)-(1.3). Our approach is to start from a bistable diffusion-only system $(\Gamma=0)$ and see how including diffraction will affect its bistable responses. Therefore, our study models the physics of systems such as InSb etalons, in which diffusion is the dominant transverse effect [11].

\subsection{Diffusive bistability}

The steady states of a diffusion-only system $(\Gamma=0)$ satisfy a scalar ordinary differential equation:

$$
\frac{\mathrm{d}^{2}}{\mathrm{~d} x^{2}} h(x)-h(x)+\left(1+r_{\mathrm{b}}^{2}\right) f(\delta+2 h(x)) I_{\text {in }}(x)=0 .
$$

When the input profile $I_{\text {in }}(x)$ is a $\delta$-function, an analytic form of the Poincare map of this ordinary differential equation can be obtained. With this map, bistability can be found analytically. But this $\delta$-function assumption ignores the shape of the input profile, retaining the power as only information from the input.

When the input has finite width, there will be no closed form for the Poincaré map. But if the input is relatively narrow, the solution of (4.1) should be close to the one from the map. So the map can be used as a guideline in selecting parameters, and the solution of the map can be used as a starting point to find bistable solutions for the diffusion-only system.

A Gaussian profile,

$$
F_{\text {in }}(x)=\sqrt{\frac{P_{\text {in }}}{\pi w^{2}}} \mathrm{e}^{-x^{2} / w^{2}},
$$

is used as the input for the numerical simulations. (The input surface of the cavity is assumed to be at the beam waist so that it has no phase curvature.) The power of the input, defined as

$$
P_{\text {in }}=\int\left|F_{\text {in }}(x)\right|^{2} \mathrm{~d} x
$$




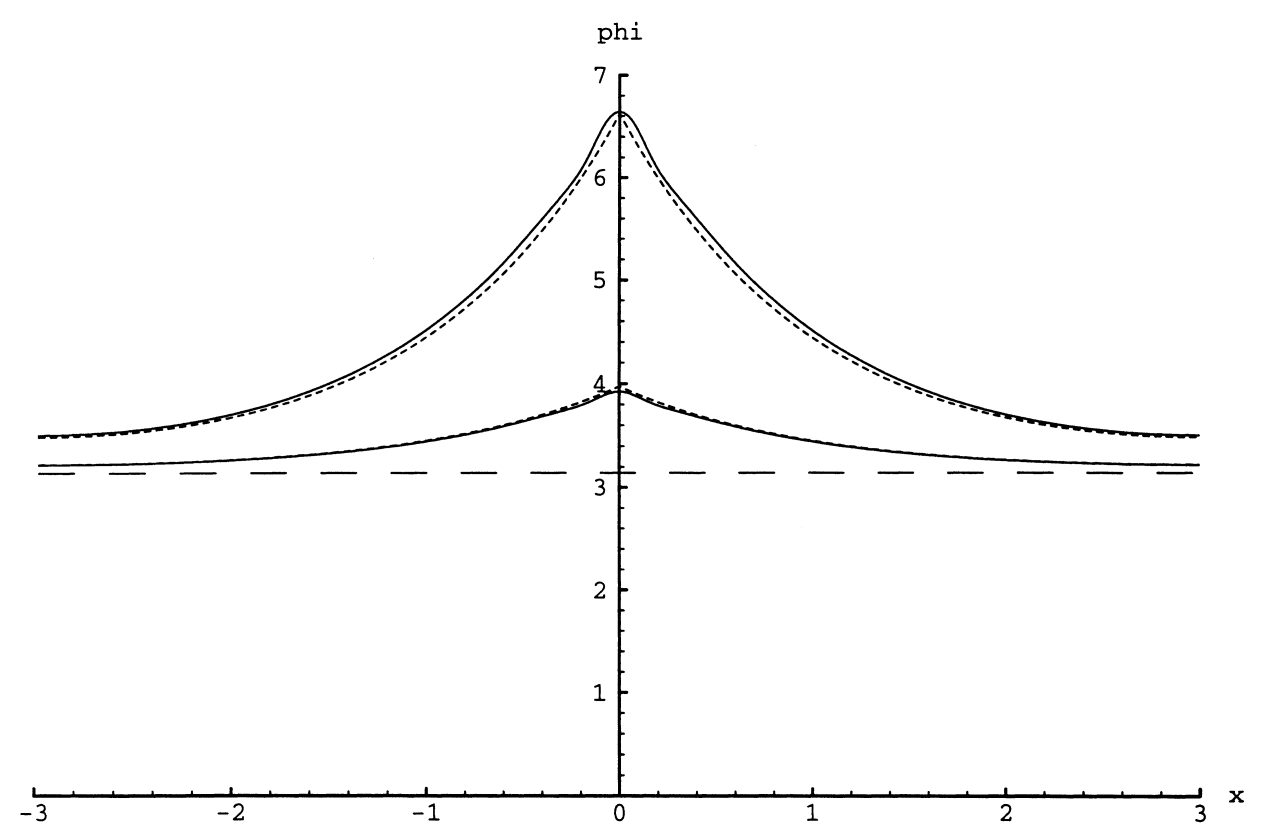

Fig. 7. Bistable solutions of a diffusive cavity with a narrow Gaussian input of width $w=0.2$. The solutions with a $\delta$-function input are shown as the dotted lines. The horizontal line corresponds to a detuning $\delta=\pi$.

is 1.125 , which is the level where bistability is found for Firth's map. The beam width, $w=0.2$, is one-fifth of the diffusion length. Fig. 7 shows the numerically computed steady states. For comparison, the solutions from the mapping theory are also shown. The major difference between the two solutions occurs at the center where solutions with finite input width are smooth, while those with infinitely narrow input have a sharp corner. Other than that, they agree quite well. The $L^{2}$ norm of the difference between them is measured to be about $2 \%$. For inputs as narrow as this, the $\delta$-function assumption is a good approximation for a diffusive system.

Once bistable solutions are found, one can examine their dependence upon beam width. Holding the power $P_{\text {in }}$ at 1.125 , we track the "on state" as the input width $w$ increases. As the width reaches 0.304 , the "on state" disappears. This can be explained by the phase shift at the beam center. When the input is narrow, the energy is concentrated in the center. That generates strong medium excitation locally, and hence a large nonlinear phase shift at the center of the beam. The phase shift is high enough that the reflected beam is in phase with the input for a large portion of the beam. For a wide beam with the same power, the energy spreads out. So the medium excitation will be low at the center and phase shift small. For a very wide beam, the medium excitation is too weak to generate the phase shift needed for the backward moving beam to be in phase with the input in any part of the beam. That will force the "on state" to switch off.

A closer examination of the data shows that at the switching point, the center phase shift of the "on state" is about 6.27. This number is very close to $2 \pi$, and is consistent with a fact from plane wave bistability that bifurcation occurs right after the phase shift reaches $2 \pi$ [2]. For the "on state" to survive, the reflected beam has to be in phase with the input, at least for a small portion of the beam.

For a wide beam, putting more energy into the system can increase center intensity, and hence increase phase shift and re-establish bistability. So stronger input is needed to maintain the "on state". Fig. 8 shows the hysteresis curves for three different input widths. The curve corresponding to a $\delta$-function input is also shown. All of them exhibit bistable behavior. But the wider the input, the more is the energy needed for bistability. In all of them, switching-off 


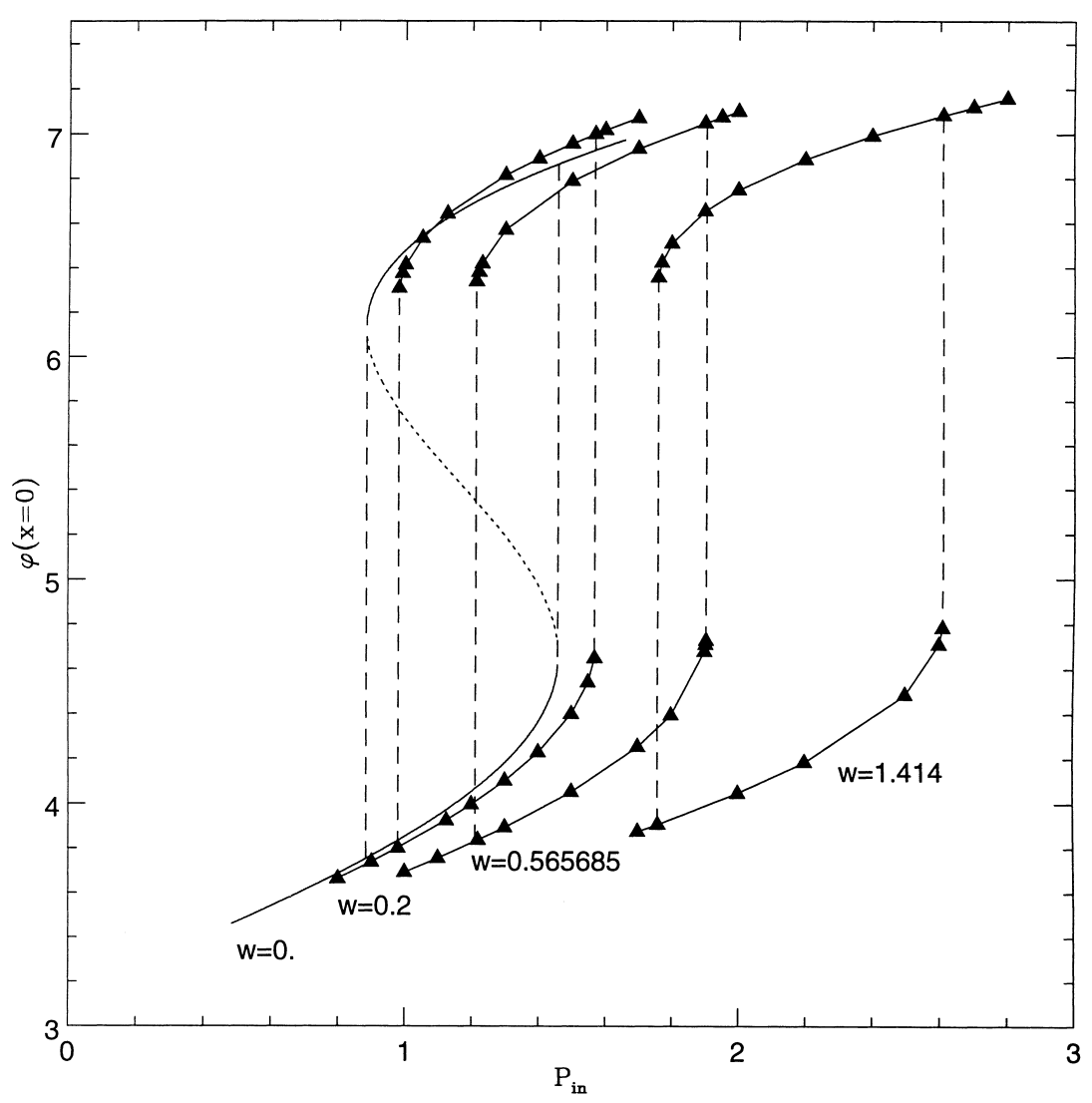

Fig. 8. Hysteresis curves of a diffusive cavity for inputs of various widths. The left-most curve corresponds to a $\delta$-function input.

occurs when the center phase shift reaches $2 \pi$. So, the center phase shift is the most important variable to be watched for switching.

\subsection{Effect of diffraction on bistability}

Diffraction causes the electromagnetic wave to broaden as it travels in a cavity. This phenomenon is characterized by forward bending wavefronts. Since diffraction preserves energy, as the beam widens its center intensity decreases. In an open space, the beam becomes weaker and weaker and eventually dies.

Nonlinearity affects the electromagnetic wave in a manner which depends on the sign of nonlinearity relative to that of diffraction. When $n_{2}$ is negative, the nonlinearity also tends to generate forward bending wavefronts, and accelerates the broadening effect due to diffraction (self-defocusing). On the other hand, when $n_{2}$ is positive, nonlinearity causes the wavefronts to bend backwards, and works against diffraction. Their interaction can produce a narrowing beam (self-focusing). In one transverse dimension, as self-focusing continues, diffraction and dispersion may reach balance and the beam settles down as a standing solitary wave. Because of its stability due to self-focusing, a solitary wave can propagate a long distance before it decays. Since the center intensity is crucial to the bistable response, focusing or defocusing nonlinearities should have very different effects on diffractive bistability. 


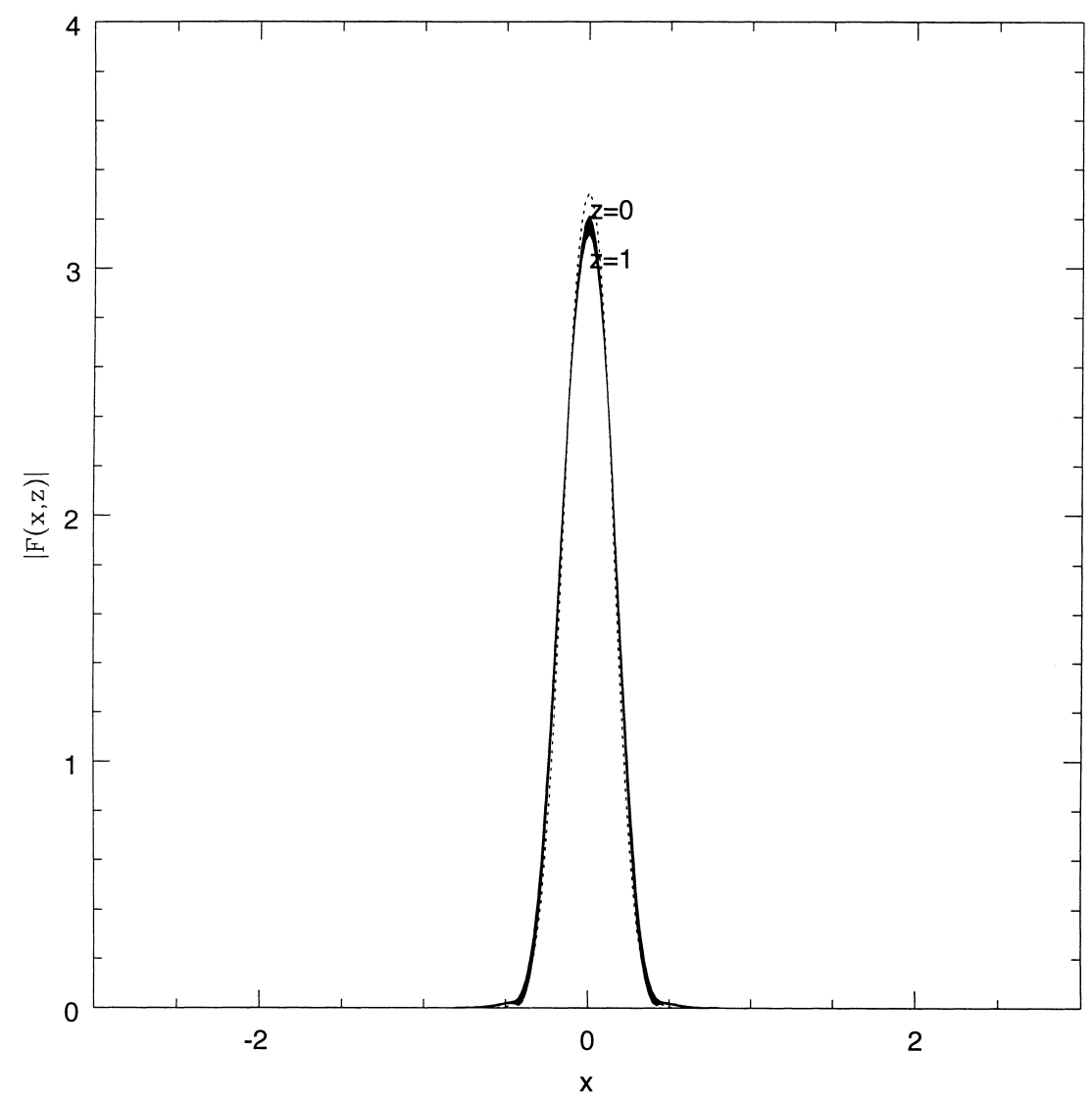

Fig. 9. The "on state" of a cavity with weak diffraction and self-defocusing nonlinearity. $\Gamma=0.001, w=0.2, \mathcal{F}=0.05$. The dotted line is the corresponding diffractionless solution $(\Gamma=0)$.

\subsubsection{Bistability with self-defocusing nonlinearity}

Fig. 9 is the result of adding a small diffraction to Fig. 7 for defocusing nonlinearity. In this figure, the forward beam is slightly wider at the back mirror than at the front. As it travels back, it widens further; and the solution at equilibrium is slightly wider than the diffractionless solution. The dotted line is the corresponding diffractionless solution $(\Gamma=0)$.

There is a small wave build up at the edge of the beam in Fig. 9. In the theory of the nonlinear Schrödinger equation, this phenomenon is called radiation. It is due to the interaction between nonlinearity and diffraction. In a free space, radiation carries energy away from the beam. In a feedback system, radiation appears in the form of secondary waves. Fig. 10 shows the details of this radiation, which moves slightly away from the center as it travels.

Fig. 11 shows the evolution of the radiation as diffraction changes. As $\Gamma$ increases, a valley starts to form. The valley reaches its deepest level at $\Gamma=0.0009$. As $\Gamma$ increases further, the valley starts to rise. It smoothes out when $\Gamma=0.0013$. During this process, the radiation wave grows stronger and eventually merges with the main wave. After the merge, the beam is wider than before the radiation is formed.

At least two more radiation waves are also observed. One occurs at $x=0.59$ around $\Gamma=0.0005$ and is on the scale of $3 \times 10^{-4}$. The other occurs at $x=0.68$ around $\Gamma=0.00038$ and its strength is only $1 \times 10^{-6}$. Both of them are further away from the main beam and much weaker than that shown in Fig. 9. 


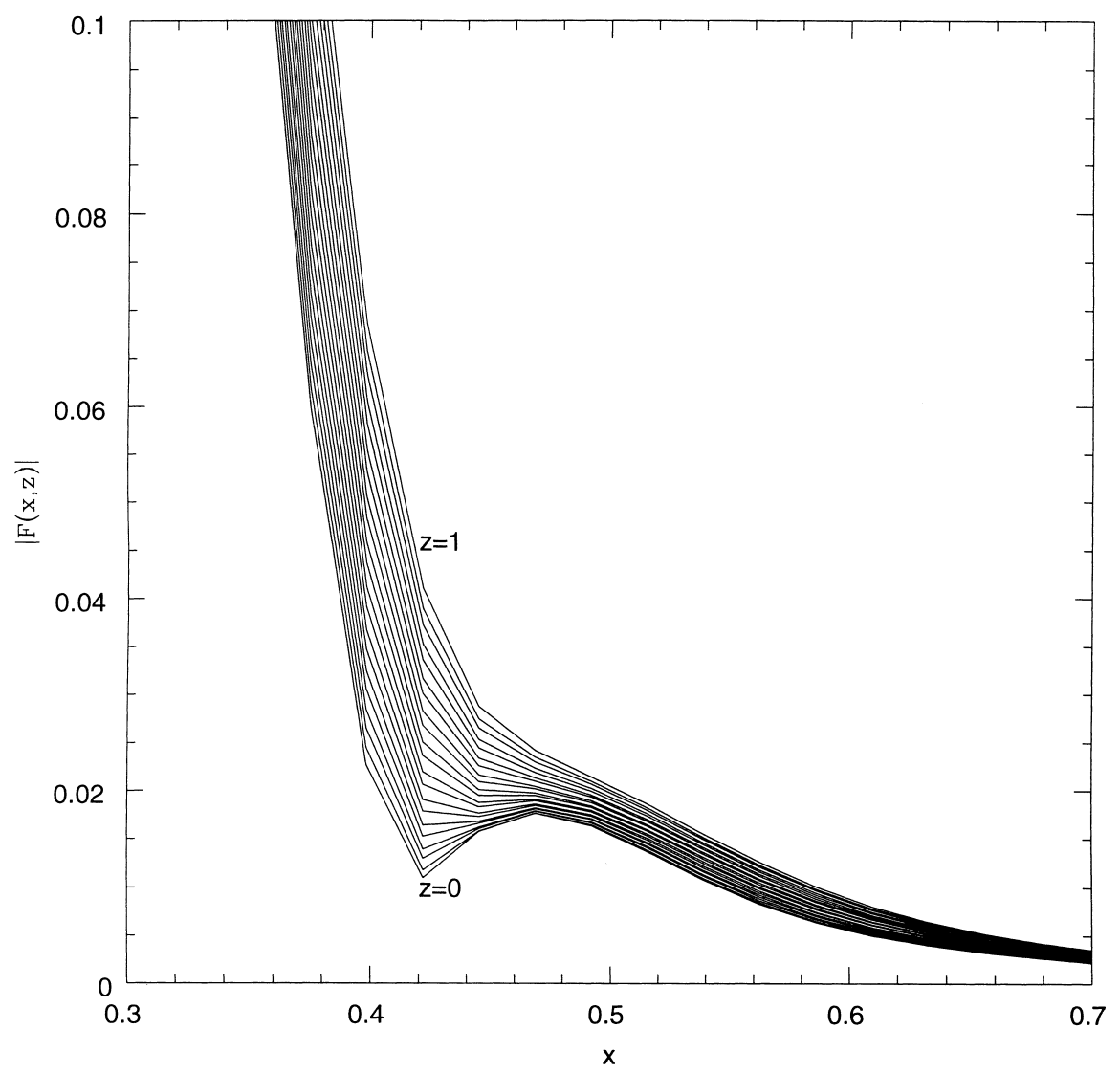

Fig. 10. The details of the radiation wave of Fig. 9.

It appears that radiation build up starts from the tail of the beam as diffraction is added to the system. Once a radiation wave is formed, it will merge with the main wave when diffraction is stronger. As soon as this wave joins the main wave, a new radiation wave will form. The new one is always closer to the center than the previous one and hence much stronger. As this process continues, the beam widens.

In a self-defocusing system, both the spreading at the center and the widening at the tail will reduce center intensity. A decrease in center intensity will affect the quality of bistability. The "left-hand side" of Fig. 12 shows the "on" and "off" states as functions of $\Gamma$ when the nonlinearity is self-defocusing. As diffraction increases, the "on" state becomes weaker and the phase shift decreases. As $\Gamma$ reaches 0.0105 , the center phase shift reaches $2 \pi$ and the "on state" disappears.

On the other hand, diffraction tends to strengthen rather than to weaken the "off state". This may be a phase effect. Because diffraction increases the center phase shift in the self-defocusing case, it makes the reflected wave less out of phase with the input. So the phase effect increases the field intensity of the "off state". When the "on" and "off" states move towards each other as diffraction increases, the bistability becomes less robust.

\subsubsection{Bistability with self-focusing nonlinearity}

Fig. 13 displays the solution for the same parameters as Fig. 9 but for self-focusing nonlinearity. The beam narrows slightly instead of spreading as it travels. Compared with the diffractionless solution, the beam profile is 


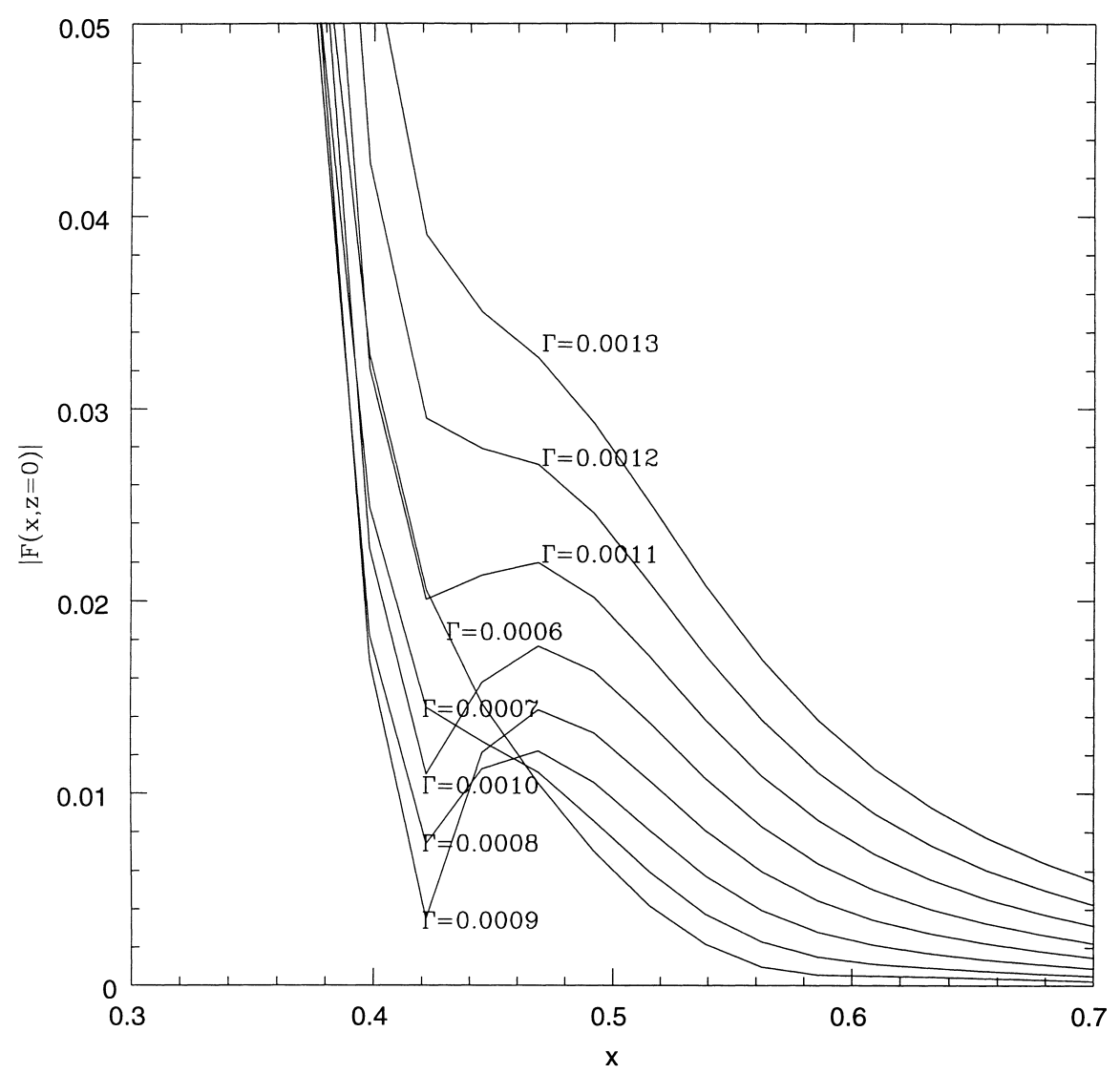

Fig. 11. The development of the radiation wave of Fig. 9 as $\Gamma$ increases.

slightly taller and narrower. Radiation waves are also observed in this case. But they are confined to a much smaller scale than in the defocusing case. For example, at $\Gamma=0.001$, the strongest radiation is on the scale of $1.5 \times 10^{-5}$ when compared to 0.015 in the self-defocusing case. Because of weaker radiation, the beam has a much cleaner tail with self-focusing nonlinearity than compared to the self-defocusing case. Since the "on state" of a self-focusing system is stronger due to energy concentration, it is more robust than in the defocusing case.

On the "right side" of Fig. 12 are the "on" and "off" states for self-focusing nonlinearity. Again "on state" strengthens as small diffraction is introduced into the system. But this process stops at $\Gamma=0.001$, where diffraction and nonlinearity balance. When $\Gamma>0.001$, diffraction dominates nonlinearity and causes the beam to broaden. Because of this, increasing diffraction further will eventually destroy bistability.

A phase effect also plays an important role in the bifurcation of the "on state". Although nonlinear refractive index of the "on state" is larger in the self-focusing case, diffraction generates a negative phase at beam center, and reduces the phase shift. Strong diffraction will eventually reduce the phase shift to a point where the reflected beam can no longer be in phase with the input. That explains why the "on state" can fail to exist, even though it is much stronger than in the self-defocusing case.

\subsubsection{Self-focusing vs. self-defocusing}

Diffraction causes the "on state" to disappear on both sides of Fig. 12. But a large difference in the separation between the "on" and "off" states at the bifurcation points is apparent. We will call this separation the "operation 

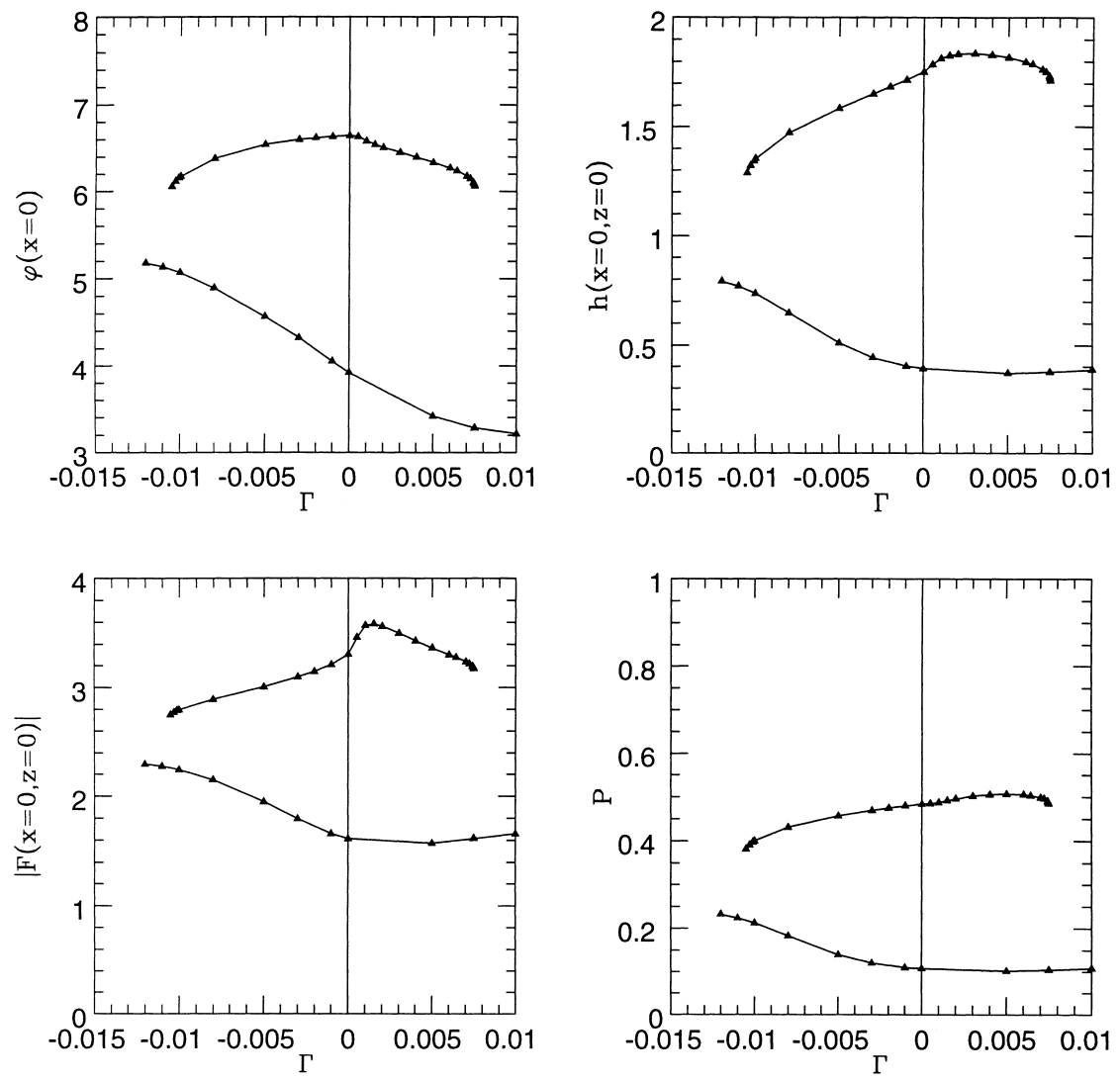

Fig. 12. Steady state solutions of a cavity for both focusing (positive $\Gamma$ ) and defocusing (negative $\Gamma$ ) nonlinearities. The upper and the lower branches correspond to the on and off states, respectively. The beam width $w=0.2$.

gap". When switching occurs in the defocusing case, the gap is very narrow, while the gap at the switching point in the focusing case is much wider. This difference results mainly from the asymmetric response of the "off state".

The asymmetry can be explained as follows. At the "off state", the back beam is out of phase with the input. If the medium is self-defocusing, diffraction will increase the phase shift and make the back beam less out of phase. That will increase the strength of the forward beam, which will further increase the phase shift. The result of this process is a taller "off state" at equilibrium when diffraction is added. The opposite happens in a focusing system. Diffraction will decrease the phase shift and make back beam more out of phase. That will reduce the forward field, which will further reduce the phase shift. So the "off state" of a focusing system is generally shorter than the diffractionless solution.

The gap or separation between the "on" and "off" states at the bifurcation (switching) point is very important to the quality of bistability. For example, one may try to maintain the bistability of a self-defocusing system when $\Gamma$ is greater than 0.001 by raising working power. With more energy into the system, the "on state" can survive stronger diffraction. But raising input power could also turn on the "off state". Because the "off state" is so close to the "on state" in a defocusing system, this can easily happen. On the other hand, the "on" and the "off" states are widely separated in a self-focusing system, so there is more freedom to raise holding power without losing the "off state". In fact, by raising holding power, we have found bistability in a self-focusing system for $\Gamma$ as high as 0.2 . While for us to find bistability in the corresponding defocusing system, $\Gamma$ had to be less than 0.04 . When $\Gamma=0.04$, the "off state" is so close to the "on state" that any increase in the input will turn it on. 


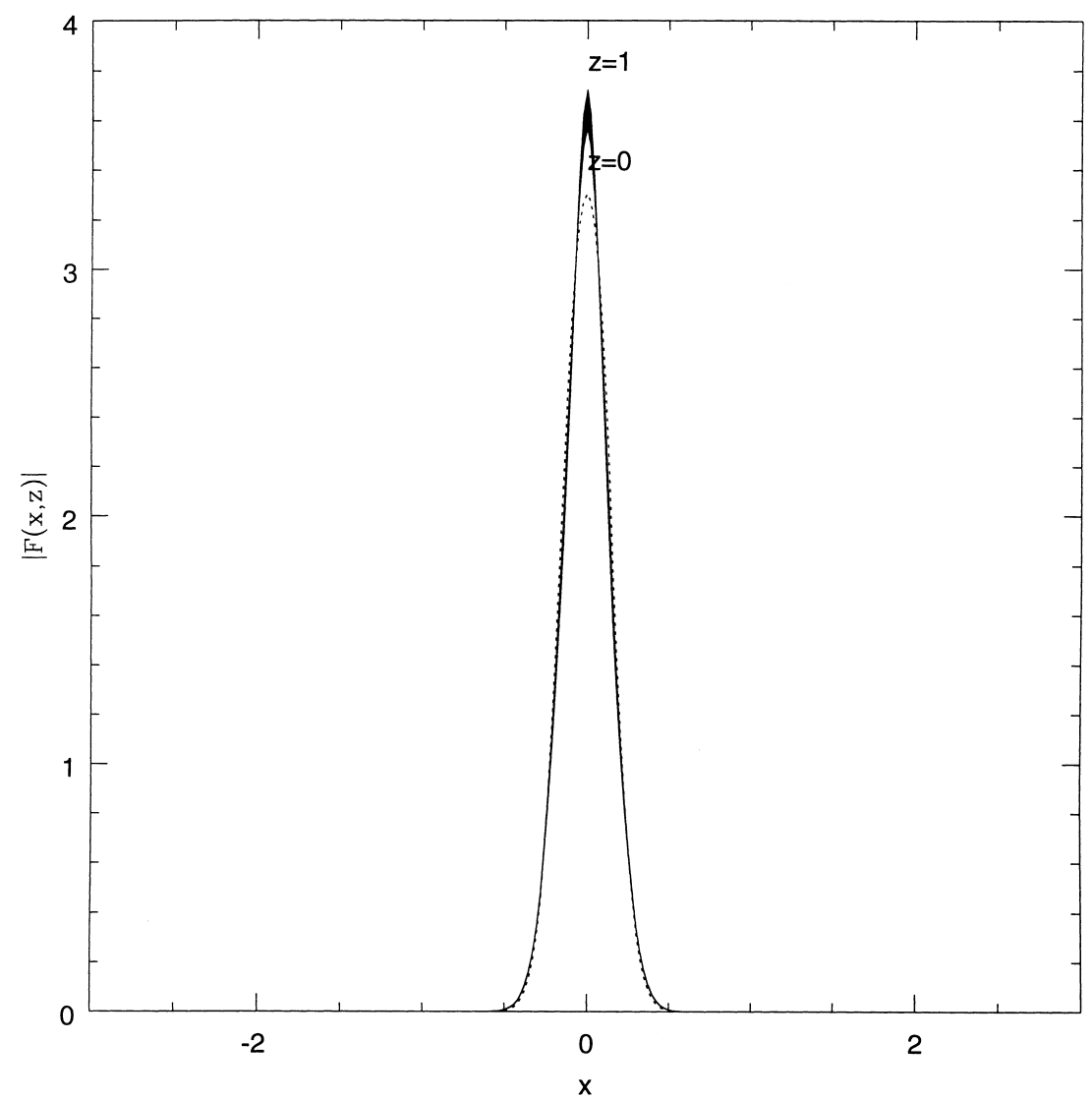

Fig. 13. The "on state" of a cavity with self-focusing nonlinearity. $\Gamma=0.001, w=0.2$. The dotted line is the corresponding diffractionless solution $(\Gamma=0)$.

A different way to compare self-focusing and self-defocusing is through hysteresis loops. Fig. 14 shows the temporal evolution of the system for both types of nonlinearity. In each case, the power $P_{\text {in }}$ of the Gaussian input is linearly ramped up from 0 to 2.5 , and then linearly ramped down to 0 . The ramping is very slow to allow the system to be close to its steady states. So the curves mimic hysteresis loops. Clearly the loop in the focusing case is much wider than the one in the defocusing case. A wider loop is preferable because the working range is wider and the switching is more robust.

A focusing medium is also superior over a defocusing one in that the hysteresis loop can survive stronger diffraction. In a self-defocusing system, the hysteresis loops become smaller and smaller and eventually disappear when diffraction is increased (Fig. 15). While in focusing system, the loop is not seen to vanish even with very high diffraction. This is consistent with an earlier observation that the gap between the "on" and the "off" states is much narrower in a defocusing system than in a focusing system.

\subsection{Soliton solutions as "on states"}

As described in detail above, bistability is robust with self-focusing nonlinearity. This is to be expected because self-focusing will cause energy concentration and narrowing of the beam's intensity profile. In a self-focusing nonlinear Schrödinger equation, this energy concentration may saturate in solitary waves which, because they do 


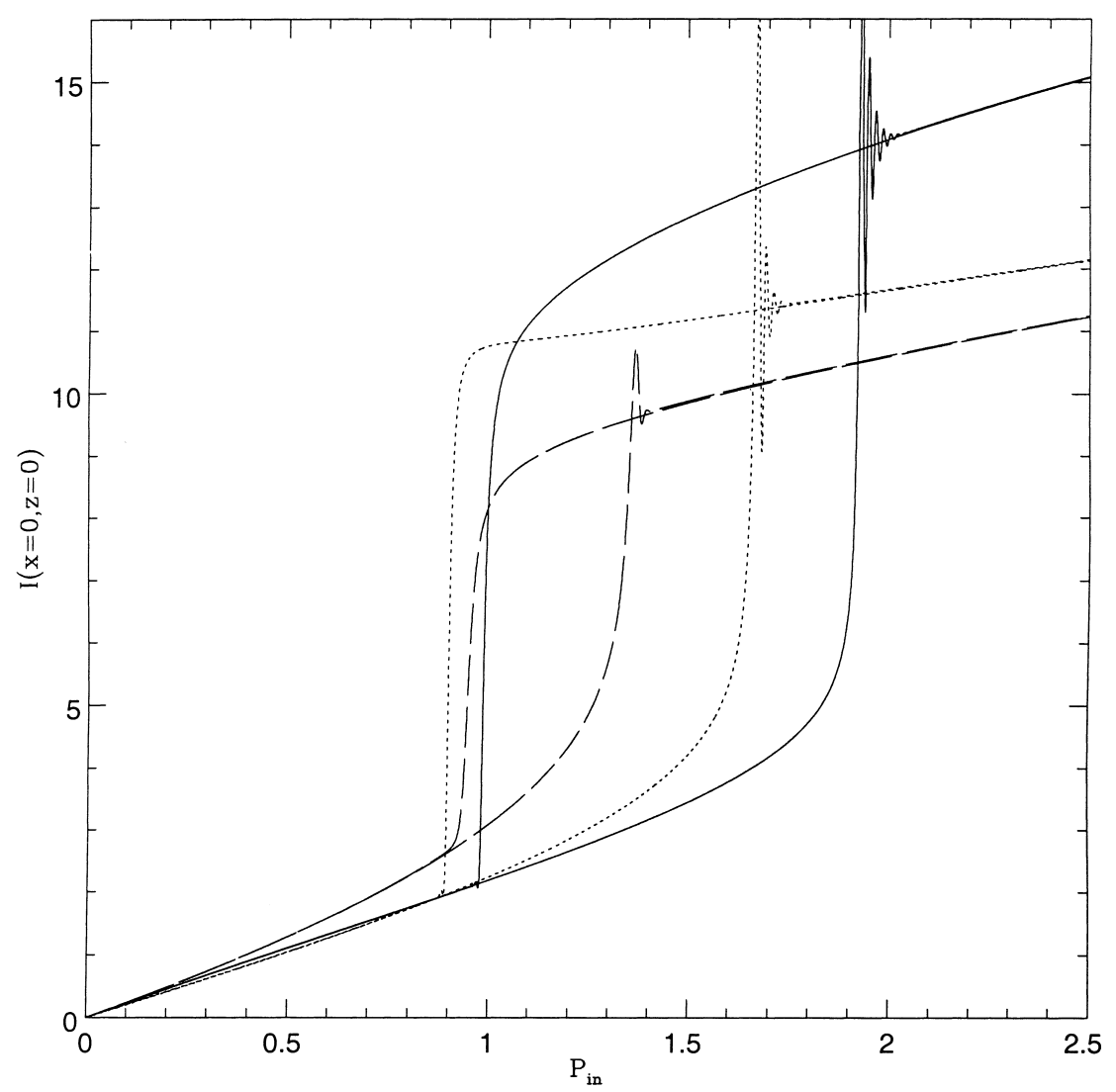

Fig. 14. Adiabatic solutions which mimic hysteresis loops. In each case, the power $P_{\text {in }}$ of the Gaussian input is linearly (in time) ramped up from 0 to 2.5 during a time interval of 500 round trips and then linearly ramped down to zero during the same period of time. The solid line has self-focusing nonlinearity and dashed line has self-defocusing nonlinearity, both with $\Gamma=0.005$. The dotted line is diffractionless.

not spread, are ideal candidates for "on" states or pixels of an all-optical array. This idea was proposed in late 1980s and studied by McDonald and Firth [13,14] for a diffraction-only system.

However, soliton-like solutions are not seen in our numerical results. Moreover, focusing is seen only for very small diffraction. When $\Gamma>0.001$, the beam broadens even in a self-focusing system. All of these suggest that the nonlinearity in our system must effectively be very weak that it can only balance little diffraction.

\subsection{Weakness of nonlinearity}

The weakness of nonlinearity can be explained mathematically. For this purpose, it will suffice to consider unidirectional propagation. The intuition can equally apply to a feedback system. Thus, in our model, we set $B$ to zero. The equations for steady states are:

$$
\begin{aligned}
& \frac{\partial F}{\partial z}-\mathrm{i} \Gamma \frac{\partial^{2}}{\partial x^{2}} F=\mathrm{i} h F, \\
& l_{\mathrm{D}}^{2} \frac{\partial^{2}}{\partial x^{2}} h-h+|F|^{2}=0 .
\end{aligned}
$$

(A diffusion coefficient is included so that the strength of the diffusive effect on nonlinearity can be addressed.) 


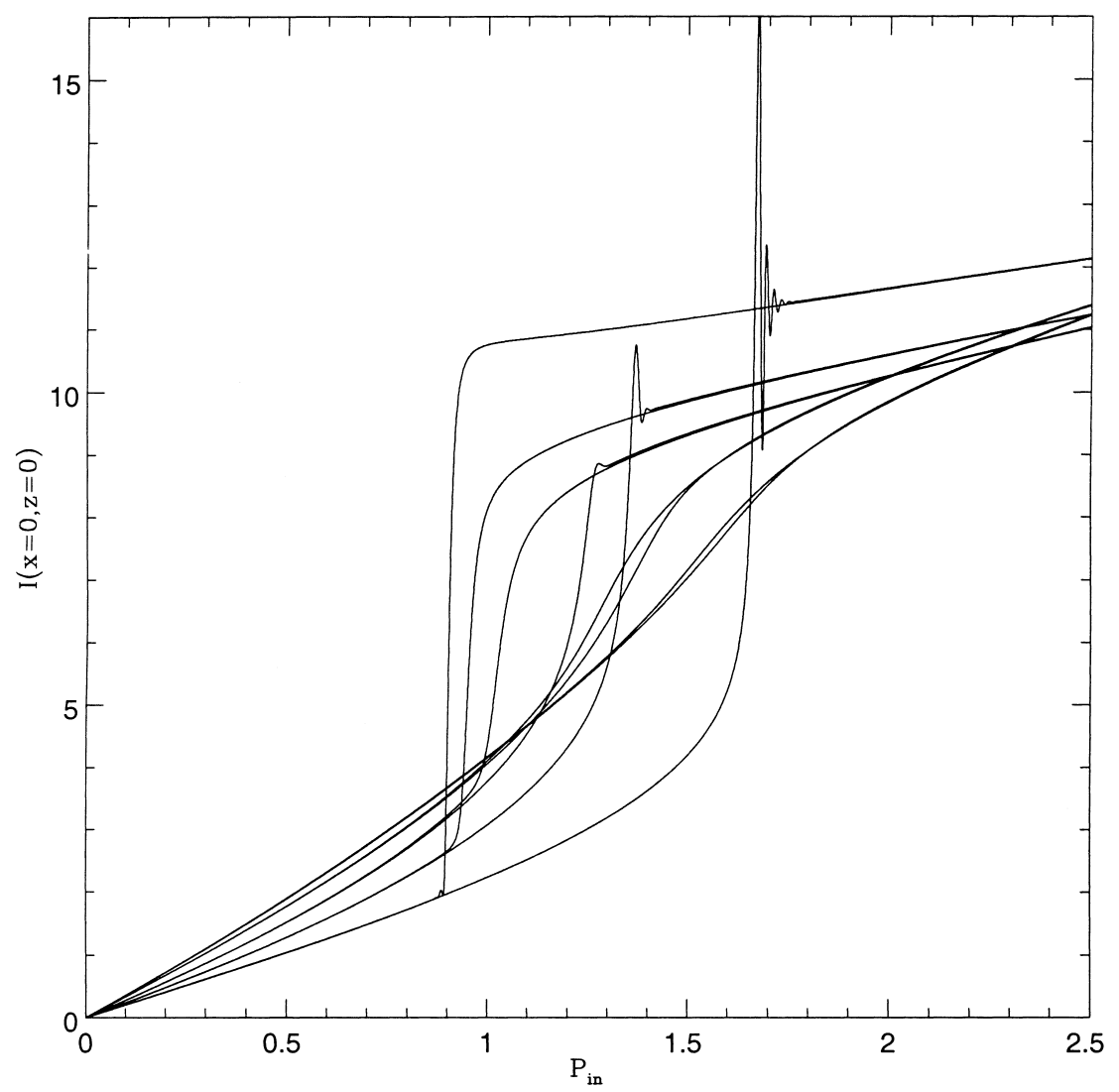

Fig. 15. Hysteresis loop vanishes in a self-defocusing system if diffraction is too strong. The widest loop corresponds to $\Gamma=0$ and the diminishing one corresponds to $\Gamma=0.05$. The rest are for $\Gamma=0.005,0.01$ and 0.03 , respectively.

The solution of (4.3) can be written as

$$
h(x)=\int_{-\infty}^{+\infty} G\left(x-x^{\prime}\right)\left|F\left(x^{\prime}\right)\right|^{2} \mathrm{~d} x^{\prime}
$$

where $G(x)=\left(1 / 2 l_{\mathrm{D}}\right) \mathrm{e}^{-|x| / l_{\mathrm{D}}}$. With this, $h$ can be eliminated from (4.2):

$$
\frac{\partial F}{\partial z}-\mathrm{i} \Gamma \frac{\partial^{2}}{\partial x^{2}} F=\mathrm{i}\left[\int_{-\infty}^{+\infty} G\left(x-x^{\prime}\right)\left|F\left(x^{\prime}\right)\right|^{2} \mathrm{~d} x^{\prime}\right] F .
$$

In this form, the nonlinearity becomes more explicit. It is cubic as a nonlinear Schrödinger equation; however, the integral on the right-hand side of (4.4) makes the nonlinearity non-local and smoothing. This integral determines the strength of the nonlinearity. Since it is the convolution of a Green's function with the intensity of the electromagnetic beam, the width of the Green's function relative to that of the beam is crucial. The width of the Green's function is measured by $l_{\mathrm{D}}$, while the width of the input provides a measure of the beam width. The "classic" nonlinear Schrödinger equation corresponds to the limit $l_{\mathrm{D}} \rightarrow 0$ and represents the strongest nonlinearity. At this limit, $G(x)$ is approaching a $\delta$-function and the nonlinearity is purely local.

In the other extreme, $l_{\mathrm{D}} \gg w$, then $G(x)$ is broad compared to the beam width, and the result of the convolution will also be broad compared to intensity profile. So the intergal will essentially be a constant within 


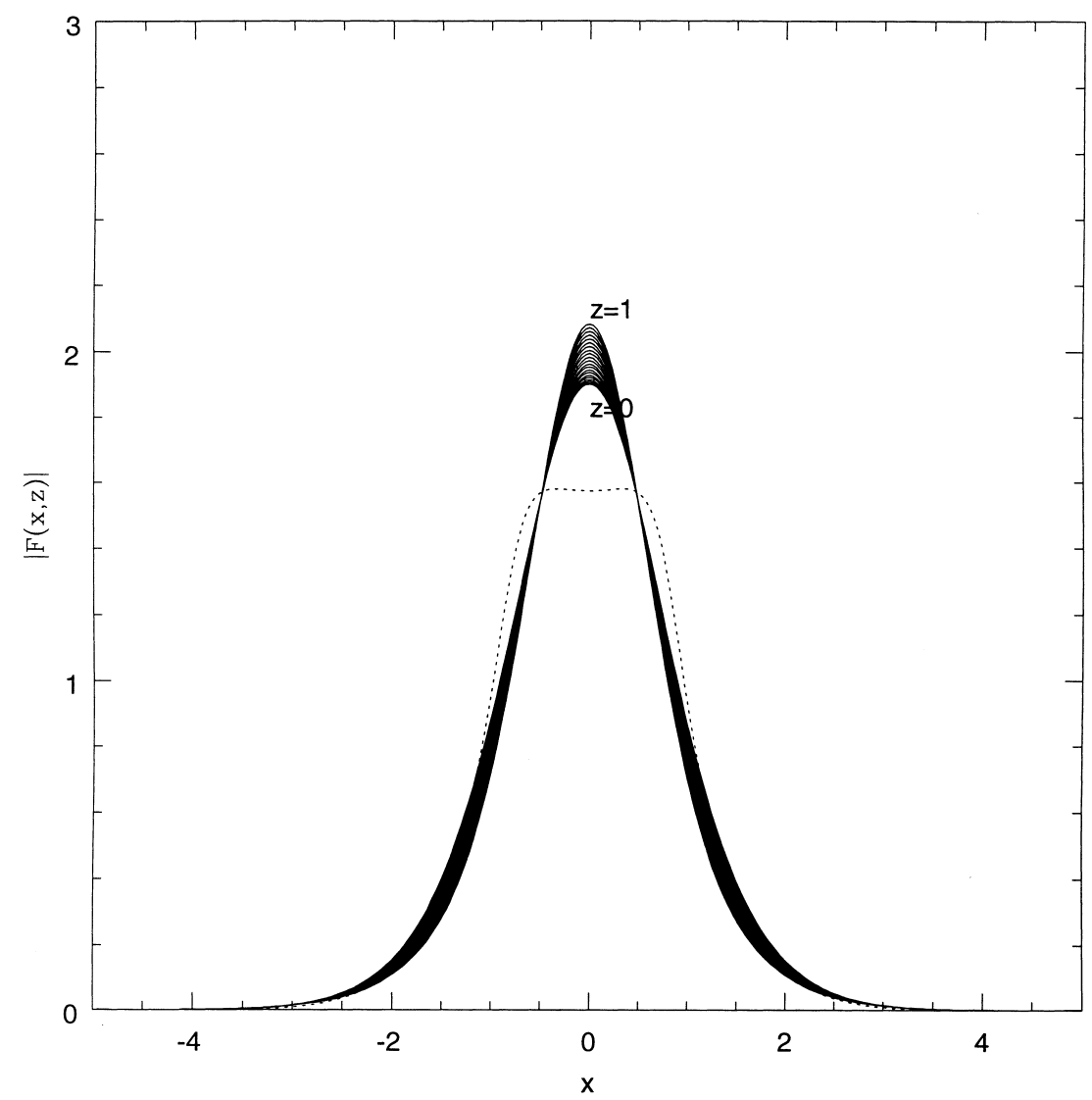

Fig. 16. The "on state" of a self-focusing cavity with a wide Gaussian input: $F_{\text {in }}=\sqrt{P_{\text {in }} / \pi w^{2}} \mathrm{e}^{-x^{2} / w^{2}}$, where the width $w=1.414$ and the power $P_{\text {in }}=2.20 . \Gamma=0.02$. The dotted line corresponds to a diffractionless solution $(\Gamma=0)$.

the beam width. At this limit, (4.4) is almost linear and resembles a diffraction equation. For the numerics of this section, $l_{\mathrm{D}}=5 w$, which is effectively close to this limit. This explains why strong focusing is not observed.

To verify this argument, an input with width $w=1.414$ is used in Fig. 16. This beam width is on the same scale as the diffusion length $\left(l_{\mathrm{D}}=1\right)$. Compared with Fig. 13, focusing is clearly stronger but still far weaker than in a nonlinear Schrödinger equation.

Fig. 16 suggests that to see strong focusing, diffusion has to be very weak. To test this, instead of letting $w$ vary, we fix $w$ and let diffusion vary. Fig. 17 shows the results for four different levels of diffusion $\left(D=l_{\mathrm{D}}^{2}\right)$. It is clear that focusing is stronger when $D$ is smaller. But it is only when $D$ is as small as 0.1 that the focusing is strong enough to greatly affect the shape of the beam. In other words, strong focusing occurs only when the width of a laser beam is significantly larger than the diffusion length.

Can the shape of the input profile affect focusing behavior? This question is inspired by the fact that a soliton has a profile of a sech function instead of a Gaussian, which leads to a suspicion that an input with a sech profile might be more adapted to a focusing system. In Fig. 17, a sech function is used as the input profile. At $D=1$, the focusing is essentially the same as earlier numerical experiment with Gaussian input [2,3]. Shape does not seem to be an important factor in diffusion dominated systems. 

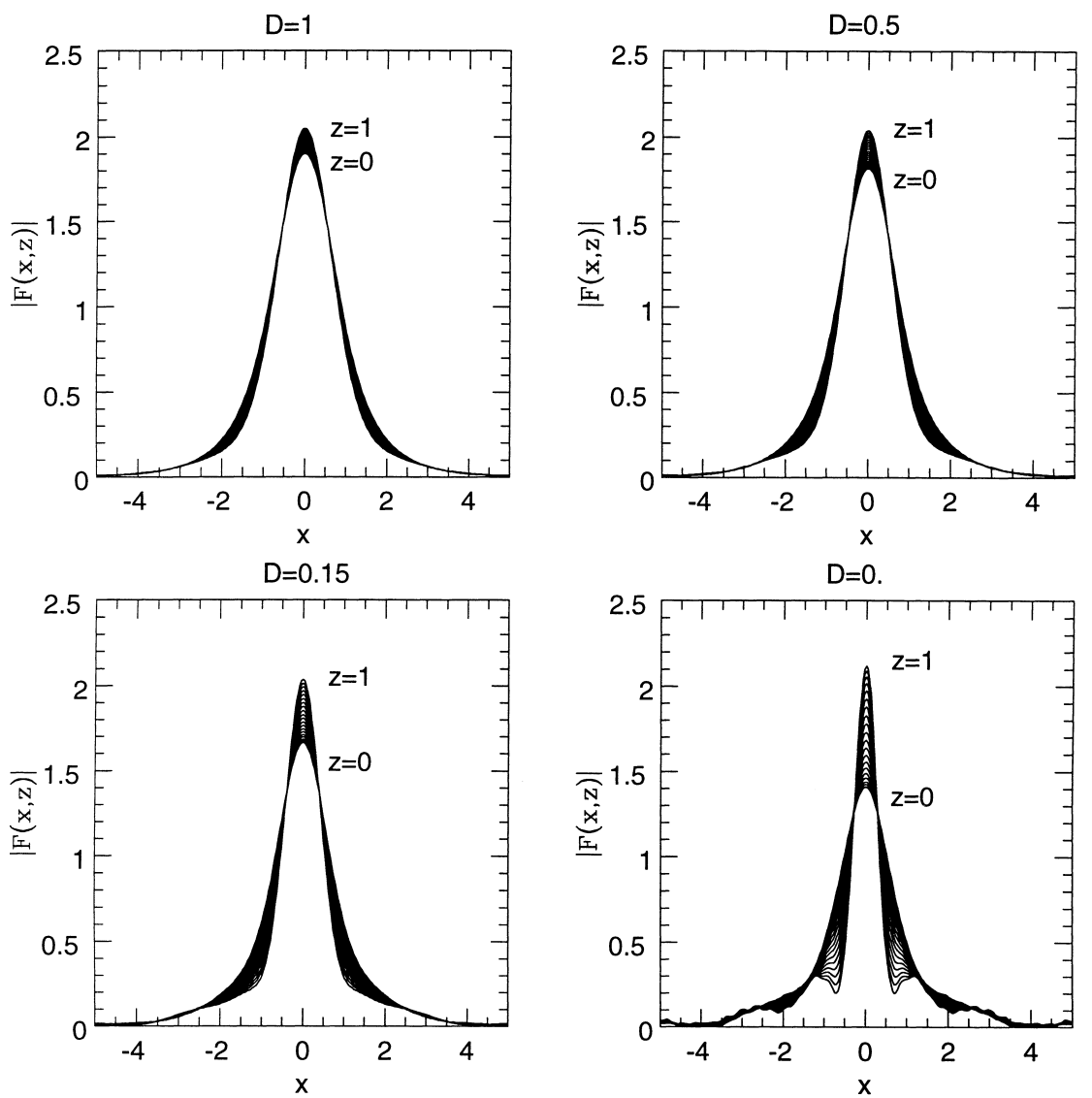

Fig. 17. Solutions of a self-focusing cavity for different levels of diffusion. The input is distributed as a sech function: $F_{\text {in }}=\sqrt{P_{\text {in }} / 2 w} \operatorname{sech}(x / w)$, where the width $w=0.9$ and the power $P_{\text {in }}=2.20 . \Gamma=0.02$ in all curves.

\subsection{Wide input}

Nonlinear effects are stronger for wider beams. This has been demonstrated by stronger focusing in Fig. 16 where the nonlinearity is self-focusing. Fig. 18 is the solution with the same input as Fig. 16, but with self-defocusing nonlinearity. The differences between these wide beam cases is much more obvious than the difference between Fig. 13 and Fig. 9 where the input beams are narrow. The field spreads strongly in Fig. 18, hence its intensity is much weaker than in Fig. 16 where it focuses. There is also a big difference in the tails. While Fig. 16 has a very smooth tail, the tail in Fig. 18 is much wider and contains strong radiation waves.

Even though focusing is not very strong when input is narrow, it has a significant impact on the quality of bistability. When input is wider, this effect will be even greater. On the other hand, stronger spreading and radiation will strongly reduce the quality of self-defocusing stability. So the wider the input is, the more important is the sign of nonlinearity. Fig. 19 shows the same curves as Fig. 12, but for an input beam with width $w=1.414$. The difference between different signs of nonlinearity is much greater than in Fig. 12 where $w=0.2$. A self-focusing medium is even more favorable for a desirable device when input is wider. 


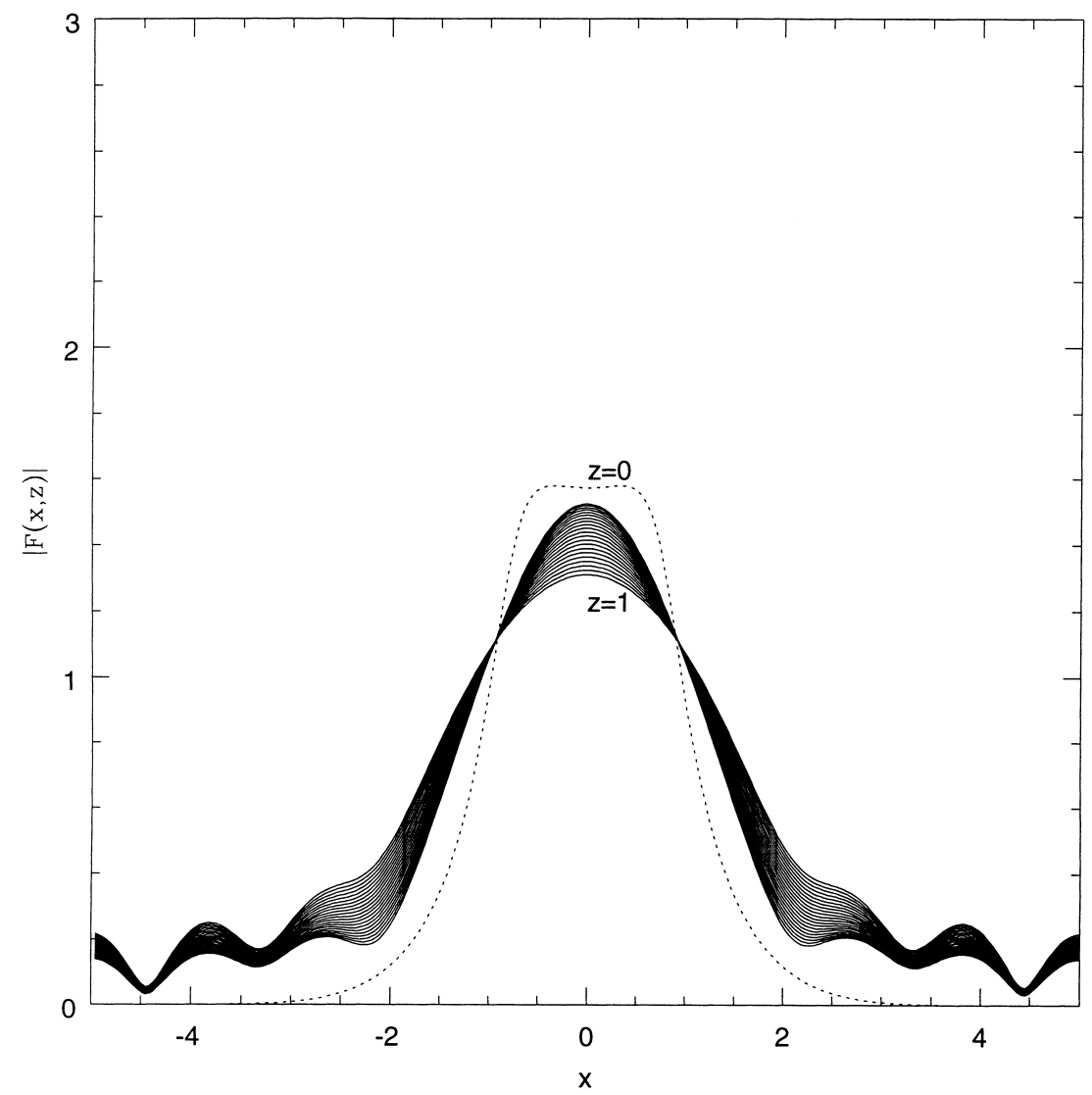

Fig. 18. The "on state" of a self-defocusing cavity with a wide Gaussian input. The beam width $w=1.414 . P_{\text {in }}=2.20, \Gamma=0.02$. The dotted line corresponds to a diffractionless solution $(\Gamma=0)$.

\section{An approximation for narrow inputs}

As discussed in Section 4.4, for narrow inputs, system (1.1)-(1.3) is weakly nonlinear. In this situation, we reduce the full system to a discrete map. This reduction takes place in two steps: first, we approximate the wave equations, and then the media equation.

\subsection{Approximating the wave equations}

The steady states of the full model satisfy

$$
\frac{\partial F}{\partial z}-\mathrm{i} \Gamma \frac{\partial^{2}}{\partial x^{2}} F=\mathrm{i} h F, \quad \frac{\partial B}{\partial z}-\mathrm{i} \Gamma \frac{\partial^{2}}{\partial x^{2}} B=\mathrm{i} h B, \quad \frac{\partial^{2}}{\partial x^{2}} h-h+\left(|F|^{2}+|B|^{2}\right)=0 .
$$

The carrier density $h(x, z)$ does not vary much across a laser beam when the beam's width is much smaller than the diffusion length. Hence, for narrow input we can treat $h$ as a constant in the wave equations for each laser beam. We denote this constant by $h_{n}$, where the subscript $n$ refers to the $n$th pixel. With this approximation, the wave equations become linear: 

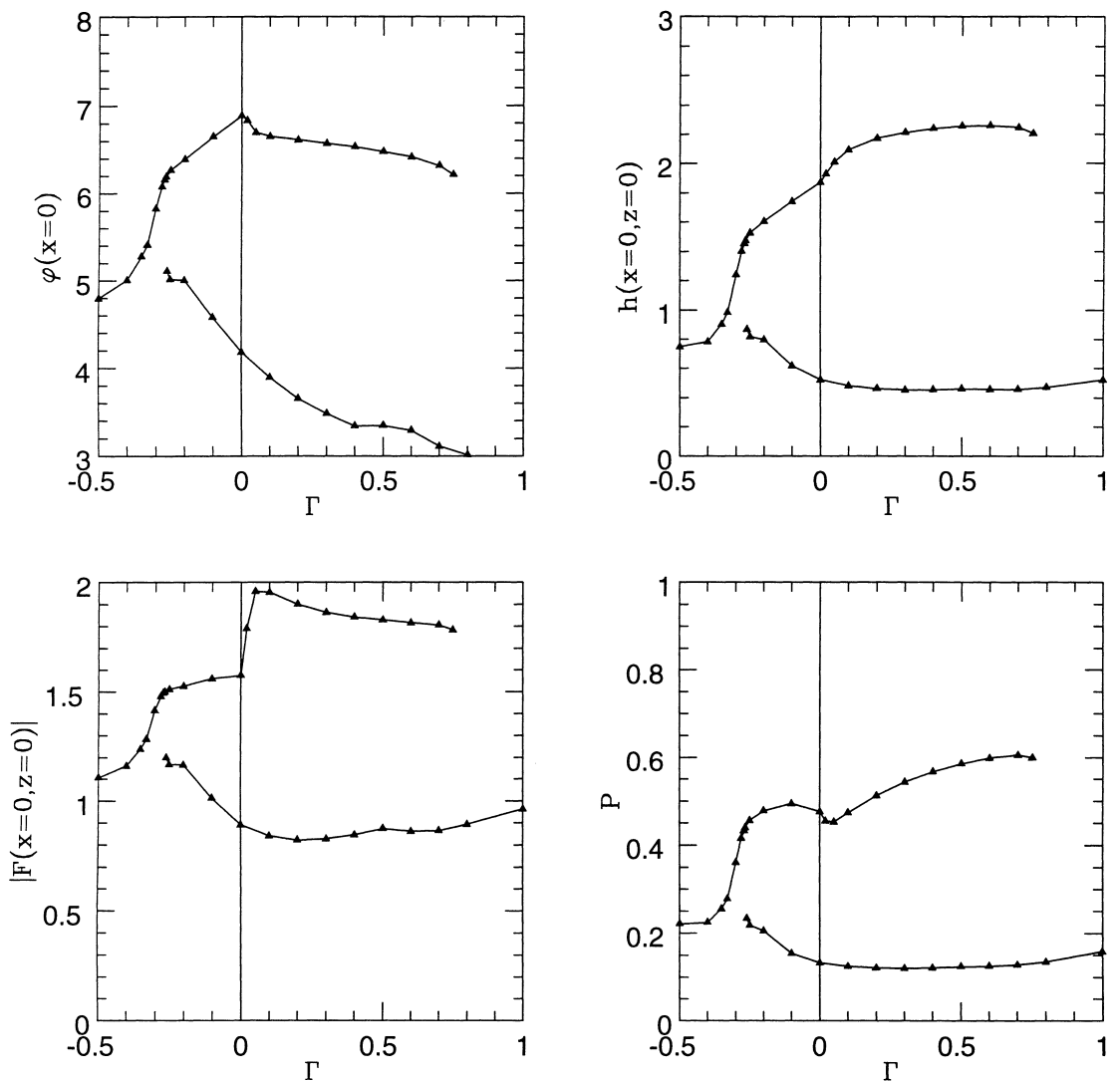

Fig. 19. Bistable solutions as functions of diffraction for a cavity with a wide input. The beam width $w=1.414$.

$$
\frac{\partial F}{\partial z}-\mathrm{i} \Gamma \frac{\partial^{2}}{\partial x^{2}} F=\mathrm{i} h_{n} F, \quad \frac{\partial B}{\partial z}-\mathrm{i} \Gamma \frac{\partial^{2}}{\partial x^{2}} B=\mathrm{i} h_{n} B,
$$

and can be solved explicitly:

$$
F_{h_{n}}(x, z)=\int \mathrm{e}^{\mathrm{i} h_{n} z} K(x-u, z) F_{h_{n}}(u, 0) \mathrm{d} u, \quad B_{h_{n}}(x, z)=\int \mathrm{e}^{\mathrm{i} h_{n}(1-z)} K(x-u, 1-z) B_{h_{n}}(u, 1) \mathrm{d} u,
$$

where $K(x, z)$ is the Green's function of the diffraction equation. The solutions are subscripted with $h_{n}$ to emphasize their dependence on this constant.

From the boundary conditions, we can write

$$
F_{h_{n}}(x, 0)=F_{\text {in }}(x)+\int r \mathrm{e}^{\mathrm{i}\left(\delta+2 h_{n}\right)} K(x-u, 2) F_{h_{n}}(u, 0) \mathrm{d} u .
$$

Iterating this integral equation yields

$$
F_{h_{n}}(x, 0)=\sum_{k=0}^{+\infty} \int\left(r \mathrm{e}^{\mathrm{i}\left(\delta+2 h_{n}\right)}\right)^{k} K(x-u, 2 k) F_{\text {in }}(u) \mathrm{d} u .
$$


Now the solutions can be written explicitly

$$
\begin{aligned}
& F_{h_{n}}(x, z)=\sum_{k=0}^{+\infty} \int\left(r \mathrm{e}^{\mathrm{i} \delta}\right)^{k} \mathrm{e}^{\mathrm{i} h_{n}(2 k+z)} K(x-u, 2 k+z) F_{\text {in }}(u) \mathrm{d} u, \\
& B_{h_{n}}(x, z)=r_{\mathrm{b}} \mathrm{e}^{\mathrm{i} \delta} \sum_{k=0}^{+\infty} \int\left(r \mathrm{e}^{\mathrm{i} \delta}\right)^{k} \mathrm{e}^{\mathrm{i} h_{n}(2 k+2-z)} K(x-u, 2 k+2-z) F_{\text {in }}(u) \mathrm{d} u .
\end{aligned}
$$

Notice that the $k$ th terms in each sum is simply the propagation of the input under the linear diffraction equation through $k$ round trips in the cavity. Intuitively, these solutions are infinite sums of the repeated reflections of the input within the cavity. The factor $r \mathrm{e}^{\mathrm{i} \delta}$ reflects the boundary effect on each trip and the terms involving with $\mathrm{e}^{\mathrm{i} h_{n}}$ are due to a nonlinear correction to the carrier oscillation.

In the case that the inputs are Gaussian distributed: $F_{\text {in }}(x)=\sum_{n} \sqrt{P_{0} / \pi w^{2}} \mathrm{e}^{-(x-n S)^{2} / w^{2}}$, the convolutions can be computed explicitly and the solutions are

$$
\begin{aligned}
& F_{h_{n}}(x, z)=\sqrt{\frac{P_{0}}{\pi w^{2}}} \sum_{k=0}^{+\infty} \frac{\left(r \mathrm{e}^{\mathrm{i}\left(\delta+2 h_{n}\right)}\right)^{k} \mathrm{e}^{\mathrm{i} h_{n} z}}{\sqrt{1+2 \mathrm{i} \mathcal{F}(2 k+z)}} \mathrm{e}^{-(x-n S)^{2} / w^{2}(1+2 \mathrm{i} \mathcal{F}(2 k+z))}, \\
& B_{h_{n}}(x, z)=r_{\mathrm{b}} \mathrm{e}^{\mathrm{i} \delta} \sqrt{\frac{P_{0}}{\pi w^{2}}} \sum_{k=0}^{+\infty} \frac{\left(r \mathrm{e}^{\mathrm{i}\left(\delta+2 h_{n}\right)}\right)^{k} \mathrm{e}^{\mathrm{i} h_{n}(2-z)}}{\sqrt{1+2 \mathrm{i} \mathcal{F}(2 k+2-z)}} \mathrm{e}^{-(x-n S)^{2} / w^{2}(1+2 \mathrm{i} \mathcal{F}(2 k+2-z))} .
\end{aligned}
$$

Here the Fresnel number $\mathcal{F}$ instead of $\Gamma$ is used to measure diffraction for reasons that will be clear later.

\subsection{Approximating the media equation}

Turning to the equation for the carrier density, we approximate the narrow electric fields with $\delta$-functions. That is, the medium equation can be approximated by

$$
\frac{\partial^{2}}{\partial x^{2}} h(x, z)-h(x, z)+\sum_{n} \int\left(\left|F_{h_{n}}(u, z)\right|^{2}+\left|B_{h_{n}}(u, z)\right|^{2}\right) \mathrm{d} u \delta(x-n S)=0 .
$$

Notice from (5.1) and (5.2) that the integral $\int\left(\left|F_{h_{n}}(u, z)\right|^{2}+\left|B_{h_{n}}(u, z)\right|^{2}\right) \mathrm{d} u$ is independent of $z$. If we define

$$
f_{\mathcal{F}}\left(\delta+2 h_{n}\right)=\int\left|\sum_{k=0}^{+\infty} \frac{\left(r \mathrm{e}^{\mathrm{i}\left(\delta+2 h_{n}\right)}\right)^{k}}{\sqrt{\pi w^{2}(1+4 \mathrm{i} k \mathcal{F})}} \mathrm{e}^{-u^{2} / w^{2}(1+4 \mathrm{i} k \mathcal{F})}\right|^{2} \mathrm{~d} u,
$$

then (5.3) can be written as

$$
\frac{\partial^{2}}{\partial x^{2}} h(x, z)-h(x, z)+\left(1+r_{\mathrm{b}}^{2}\right) P_{0} \sum_{n} f_{\mathcal{F}}\left(\delta+2 h_{n}\right) \delta(x-n S)=0 .
$$

The function $f_{\mathcal{F}}(\varphi)$ is subscripted with $\mathcal{F}$ to emphasize that it depends on the strength of diffraction.

To close the theory, we have to model $h_{n}$ and the model should reflect nonlinearity. Recall that $h_{n}$ is a constant that approximates $h(x, z)$ within the laser pulse at $x=x_{n}$. A natural choice for $h_{n}$ is the value of $h(x, z)$ at the center of the beam, i.e., $h_{n}=h\left(x_{n}, 0\right)$. 
Eq. (5.5) is very similar to Eq. (2.2). The only difference between them is in their nonlinearities. We may think of $f_{\mathcal{F}}(\varphi)$ as a modified characteristic function. By making this analogy, the new model is very similar to Firth's diffractionless model, and a similar analysis can be applied to (5.5). For example, (5.5) will be equivalent to a map:

$$
A_{n+1}=2 c A_{n}-s\left(1+r_{\mathrm{b}}^{2}\right) f_{\mathcal{F}}\left(\delta+2 A_{n}\right) P_{0}-B_{n}, \quad B_{n+1}=A_{n} .
$$

In particular, for a single, isolated pixel, the solution is determined by

$$
h_{0}=\frac{1}{2}\left(1+r_{\mathrm{b}}^{2}\right) f_{\mathcal{F}}\left(\delta+2 h_{0}\right) P_{0},
$$

or in terms of phase shift $\varphi_{0}=\delta+2 h_{0}$ :

$$
\varphi_{0}-\delta=\left(1+r_{\mathrm{b}}^{2}\right) f_{\mathcal{F}}\left(\varphi_{0}\right) P_{0}
$$

which can be solved graphically.

Recall that Firth's theory solved the wave equations by neglecting diffraction and then approximating the laser pulses by $\delta$-functions in the medium equation. Our theory is similar to Firth's in that it also approximates laser pulses with $\delta$-functions. But instead of ignoring diffraction, our theory approximates the refractive index in the wave equation with its value at the center of the beam. Because diffraction is not ignored, its effect on bistability can be studied.

\section{3. $f_{\mathcal{F}}(\varphi)$}

The only presence of diffraction in Eq. (5.6) is through the modified characteristic function $f_{\mathcal{F}}(\varphi)$. So diffraction can be studied through its effects on this function. Most diffractive effects can be explained by the second term in the sum of (5.4). This term has a coefficient of

$$
\frac{r \mathrm{e}^{\mathrm{i}\left(\delta+2 h_{n}\right)}}{\sqrt{1+4 \mathrm{i} \mathcal{F}}} .
$$

When $\mathcal{F}=0$ (in which case, the model reduces to Firth's), the amplitude of this coefficient is the reflectance and the phase is the nonlinear detuning. When $\mathcal{F}$ is not zero, the coefficient can be written as $r_{\mathcal{F}} \mathrm{e}^{\mathrm{i}\left(\delta_{\mathcal{F}}+2 h_{n}\right)}$, where

$$
r_{\mathcal{F}}=\frac{r}{\sqrt[4]{1+(4 \mathcal{F})^{2}}}, \quad \delta_{\mathcal{F}}=\delta-\frac{1}{2} \arctan (4 \mathcal{F})
$$

These expressions $r_{\mathcal{F}}$ and $\delta_{\mathcal{F}}$ can be viewed as effective reflectance and effective detuning, an analogy which provides intuition about diffractive effects.

Fig. 20 shows some typical plots of $f_{\mathcal{F}}(\varphi)$. Compared to the diffractionless curve, the curve shifts to the right in the focusing case and to the left in the defocusing case. These shifts can be explained easily by effective detuning. When $\mathcal{F}>0, \delta_{\mathcal{F}}<\delta$. Smaller detuning is equivalent to shifting the curve to the right. Similarly, $\delta_{\mathcal{F}}>\delta$ in the defocusing case so $f_{\mathcal{F}}(\varphi)$ shifts to the left. Generally speaking, a right shifting curve is advantageous to the "off state", because it is equivalent to a cavity with smaller detuning and hence smaller phase shift, and a left shift benefits the "on state" because the equivalent detuning is larger.

With either sign of nonlinearity, $f_{\mathcal{F}}(\varphi)$ is flattened by diffraction. This diffractive effect can be explained by effective reflectance $r_{\mathcal{F}}: r_{\mathcal{F}}<r$ for either type of nonlinearity. Smaller reflectance means a flatter characteristic curve and weaker nonlinearity. When diffraction is very strong, $f_{\mathcal{F}}(\varphi)$ can be so flat that bistability is destroyed, which explains that strong diffraction can completely destroy bistability in both focusing and defocusing cases. 


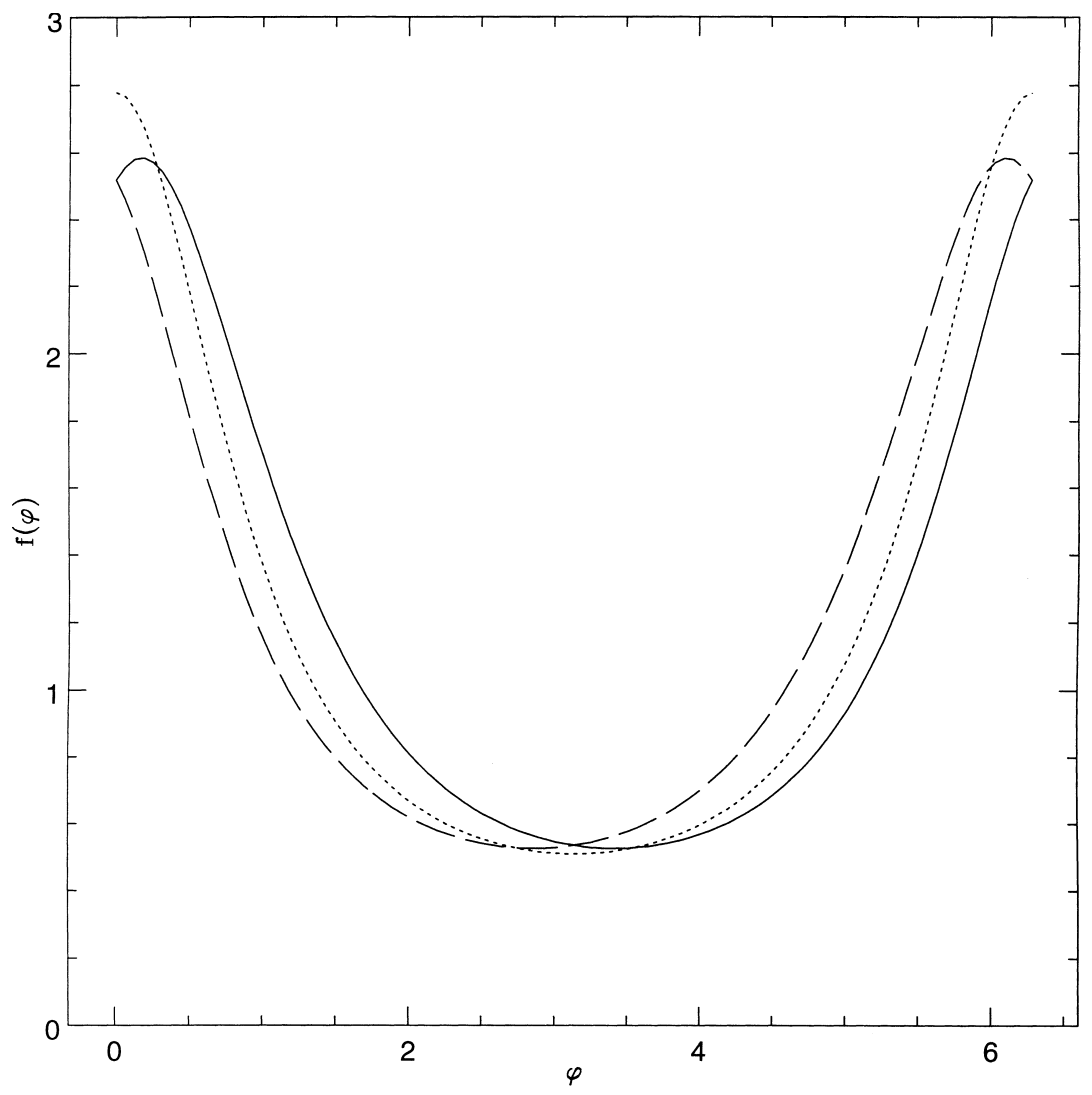

Fig. 20. $f_{\mathcal{F}}(\varphi)$ for $\mathcal{F}=0.5$. The solid line has focusing nonlinearity. The broken line has defocusing nonlinearity. The dotted line is diffractionless.

Notice that

$$
\int\left|\sum_{k=0}^{+\infty} \frac{1}{\sqrt{\pi w^{2}(1+4 \mathrm{i} k \mathcal{F})}} \mathrm{e}^{-x^{2} / w^{2}(1+4 \mathrm{i} k \mathcal{F})}\right|^{2} \mathrm{~d} x=\int\left|\sum_{k=0}^{+\infty} \frac{1}{\sqrt{\pi(1+4 \mathrm{i} k \mathcal{F})}} \mathrm{e}^{-x^{2} /(1+4 \mathrm{i} k \mathcal{F})}\right|^{2} \mathrm{~d} x .
$$

Thus, $f_{\mathcal{F}}(\varphi)$ is independent of the input width $w$. So the only dependence of $f_{\mathcal{F}}(\varphi)$ on the shape of input distribution is through $\mathcal{F}$. Once $f_{\mathcal{F}}(\varphi)$ is computed for a particular $\mathcal{F}$, it can be used for Gaussian inputs with any widths and amplitudes as long as the Fresnel number is the same. That justifies the use of $\mathcal{F}$ to label $f_{\mathcal{F}}(\varphi)$. This property implies that in this narrow beam approximation, the system's response curve to the Fresnel number will not depend on the input width.

\subsection{Bistability with the reduced model}

Eq. (5.6) is solved numerically. Fig. 21 shows the curves of Fig. 12, but as computed with this method. This figure preserves many features of Fig. 12, especially on the focusing side. For example, it shows a slight focusing on the right half plane and that the focusing stops at $\mathcal{F}=0.1$. It also shows wider gap between the "on" and "off" states on the focusing side than on the defocusing side, and it offers a reasonable estimate of the Fresnel number 

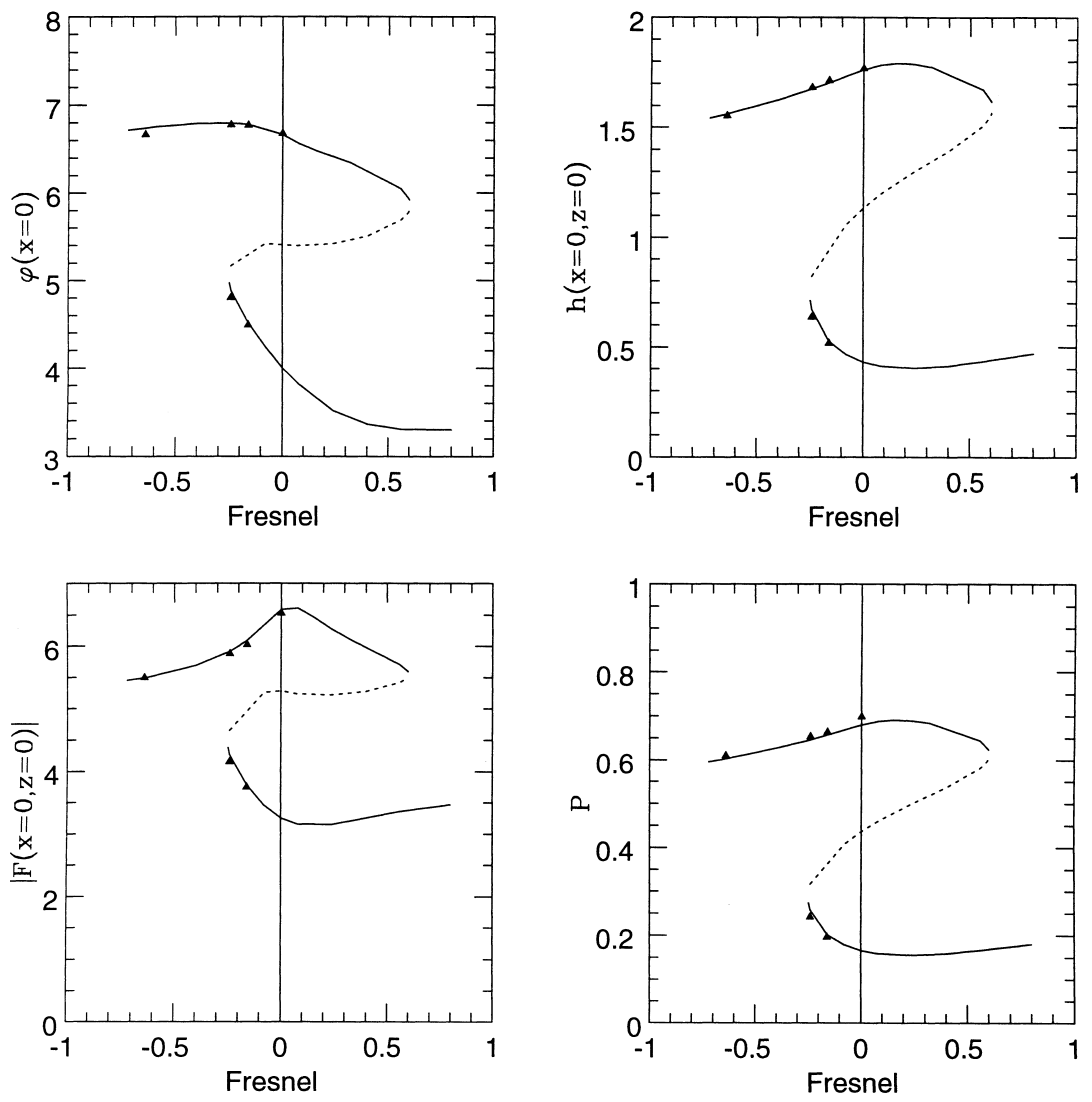

Fig. 21. Theoretical estimates for curves in Fig. 12. The marked points are numerical results for inputs with width $w=0.05$.

$(\mathcal{F}=0.57)$ at which bistability ceases to exist. However, on the defocusing side, the approximation does not agree with the precise numerical results very well.

For the input used in Fig. 12, the beam is too wide to justify the use of the approximate theory. The $h$ field varies substantially within the width of the beam, rather than being flat as required by the approximation. Computations (with the full model) for inputs narrower than $w=0.2$ were performed at the Pittsburg Supercomputing Center. We were able to compute a few points for an input of width $w=0.05$. Because our resources were insufficient to generate the full figure, we concentrated on the defocusing side where Figs. 12 and 21 do not agree. Since the theoretical response curves do not depend on input width, the same theoretical curves of Fig. 21 can be used for comparison at this narrow input. The computational results are marked in Fig. 21. They fit the theoretical curves very well. In particular, they confirm that the "off state" bifurcates before the "on state" when diffraction increases. These results strongly support the approximation theory.

Using the reduced model, the full region in which bistability exists can be estimated. This region is shown in Fig. 22. This figure proves two phenomena that were revealed by the full numerical study. One is that the gap between the "on" and the "off" states is wider on the focusing side than on the defocusing side. As $\mathcal{F}$ increases, the gap decreases on the defocusing side but increases on the focusing side. The other is that with defocusing nonlinearity, diffraction closes the gap completely. Bistability disappears when $\mathcal{F}<-0.78$. On the other hand, diffraction opens up the gap in the focusing case. Bistability exists for very large positive Fresnel number. From Fig. 22, it is obvious that the focusing nonlinearity is superior than defocusing nonlinearity for bistability. 


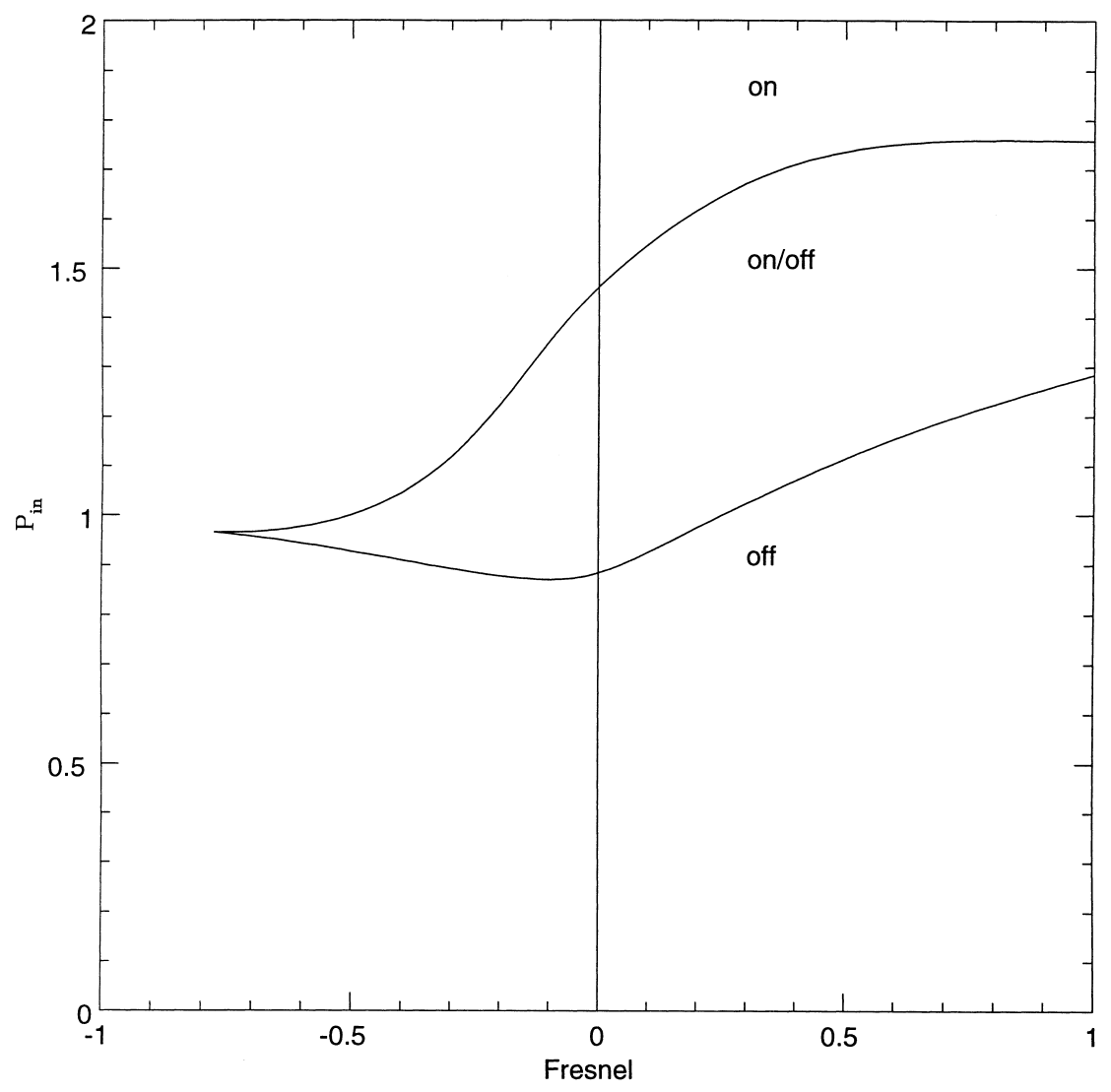

Fig. 22. The region that bistability exists, for detuning $\delta=\pi$.

\section{Optical arrays}

A single optical bistable device is only capable of storing one bit of information. However, a large number of such devices assembled into an array may form an all-optical memory for binary data. When parameters are tuned so that each individual device is operating in its bistable region, a key issue for the optical array is its packing density. The minimum pixel spacing is restricted by crosstalk between pixels. If the packing is too dense, an individual pixel within the array may not be able to be addressed independently.

Because of computational limitations, we only study arrays with a small number of pixels. In fact, we will work mostly on arrays with three pixels, which provide most of the design ingredients for larger arrays. (In fact, a numerical experiment [2] found that a three bit pattern with one "off state" embeds well within a six bit pattern, also with a single "off state".) The same strategy as in Section 4 will be followed in this section. That is, we will start with optical arrays in which diffraction is neglected. Then we will discuss how diffraction will affect packing requirements. The emphasis is again on the sign of nonlinearity. Our work, therefore, follows closely on that of Firth and Galbraith [7] which deals with bistable arrays of nonlinear FabryPérot cavities, but with an extension of the discussion to include diffraction and self-focusing-defocusing effects. 

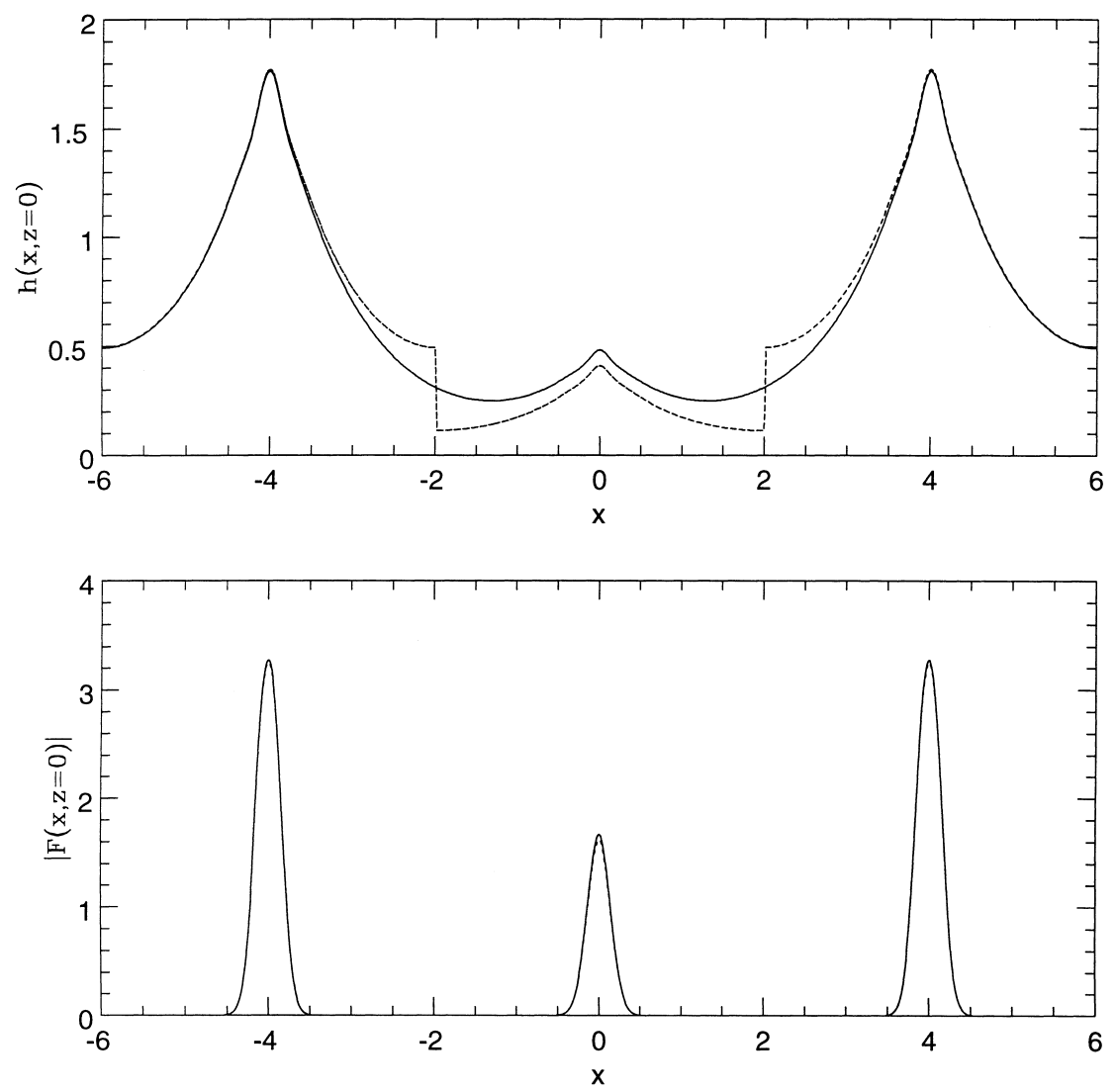

Fig. 23. The solution representing the pattern "101" of a diffusive three pixel array. The dotted line is the initial condition which is constructed by concatenating two "on state" solutions and one "off state" solution of Fig. 5. Pixel separation $S=4$.

\subsection{Diffusive optical array}

Fig. 23 shows a solution corresponding to the pattern "101", for a diffusive $(\Gamma=0)$ three pixel array with interpixel separation $S=4$. All other parameters are the same as in Fig. 5. The initial condition, shown as the dotted lines, is constructed by concatenating two "on state" solutions and one "off state" solution of Fig. 7.

In the array, the electric field of each pixel is virtually unaffected by the presence of other pixels. However the boundary of refractive index between the two "on" pixels and the "off" pixel is smoothed by diffusion. The refractive indices of the two "on" pixels are mostly unchanged locally, but their tails are pulled down on the weaker side by the "off" pixel. On the other hand, the tail of the "off" pixel are pulled up by its stronger neighbors. This creates a crosstalk between the "on" bits and the "off" bit. Physically, this phenomenon can be explained by charge carrier flow from the "on" pixels to the "off" pixel. Because of this crosstalk, the refractive index of the "off" pixel is slightly larger than when it is alone.

As packing density increases, the crosstalk will become stronger and pulls the "off" pixel further up. Fig. 24 shows this process. As $S$ reaches 2.73, the "off" pixel is turned on. This determines that the critical distance of this array is $S_{\mathrm{c}}=2.77$. This number agrees well with that of the estimation from Firth's mapping theory where the input is infinitely narrow [2]. 

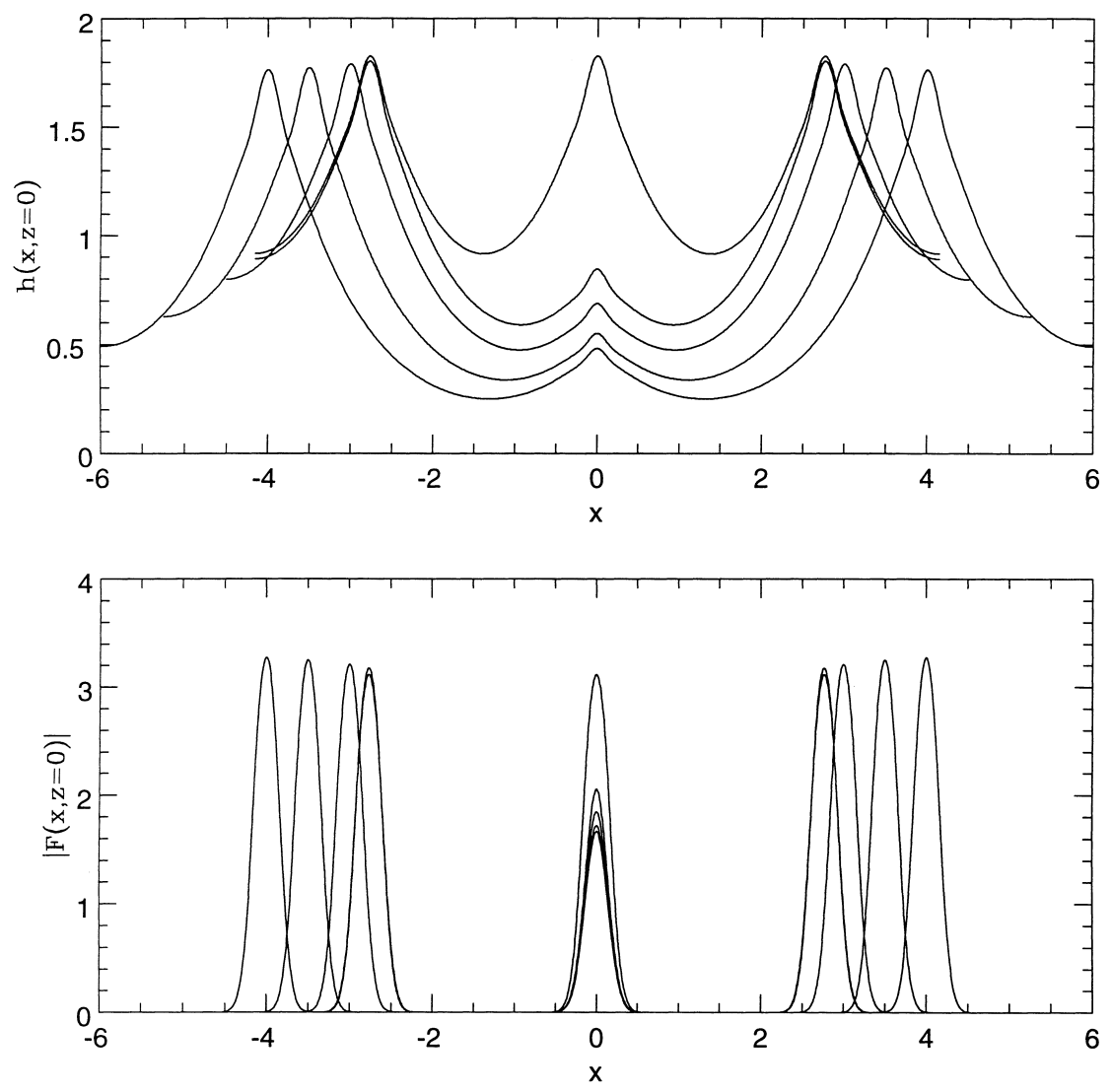

Fig. 24. The bifurcation of the pattern "101" of a diffusive three pixel array as packing density increases. Curves are for interpixel spacing of $S=4,3.5,3,2.77$ and 2.73 , respectively.

\subsection{Diffractive effect on optical arrays}

It has been shown in Section 4 that diffraction has a big impact on the quality of bistability, and that the sign of nonlinearity is critical to this impact. The difference can be measured by the "operation gap", the separation of the "on" and "off" states. In general, a bistable device with self-defocusing nonlinearity has much narrower operation gap than its counterpart with focusing nonlinearity. This means the "on" and "off" pixels are less differentiated in the defocusing case than in a focusing one.

In an array, crosstalk can cause undesired switching. Since the operation gap of self-defocusing bistability is smaller, switching is more easily induced by crosstalk in a defocusing array than in a focusing one. Our numerical results show that this switching occurs at $S=3.733$ for the same array as Fig. 24 when $\Gamma=-0.005$ (Fig. $25)$. That is significantly larger than when diffraction is neglected, where the critical distance was 2.77 . On the other hand, the distance is reduced to 2.4 if nonlinearity is self-focusing (Fig. 26). This is a $50 \%$ improvement over the self-defocusing case, and this means that for a material like InSb, the packing could increase by $50 \%$ if the nonlinearity were self-focusing. The interpixel spacing of Figs. 1 and 2 is in between the optimal spacing of the self-focusing and self-defocusing cases, which explains why switching in the defocusing array failed, while switching in the focusing array succeeded. 

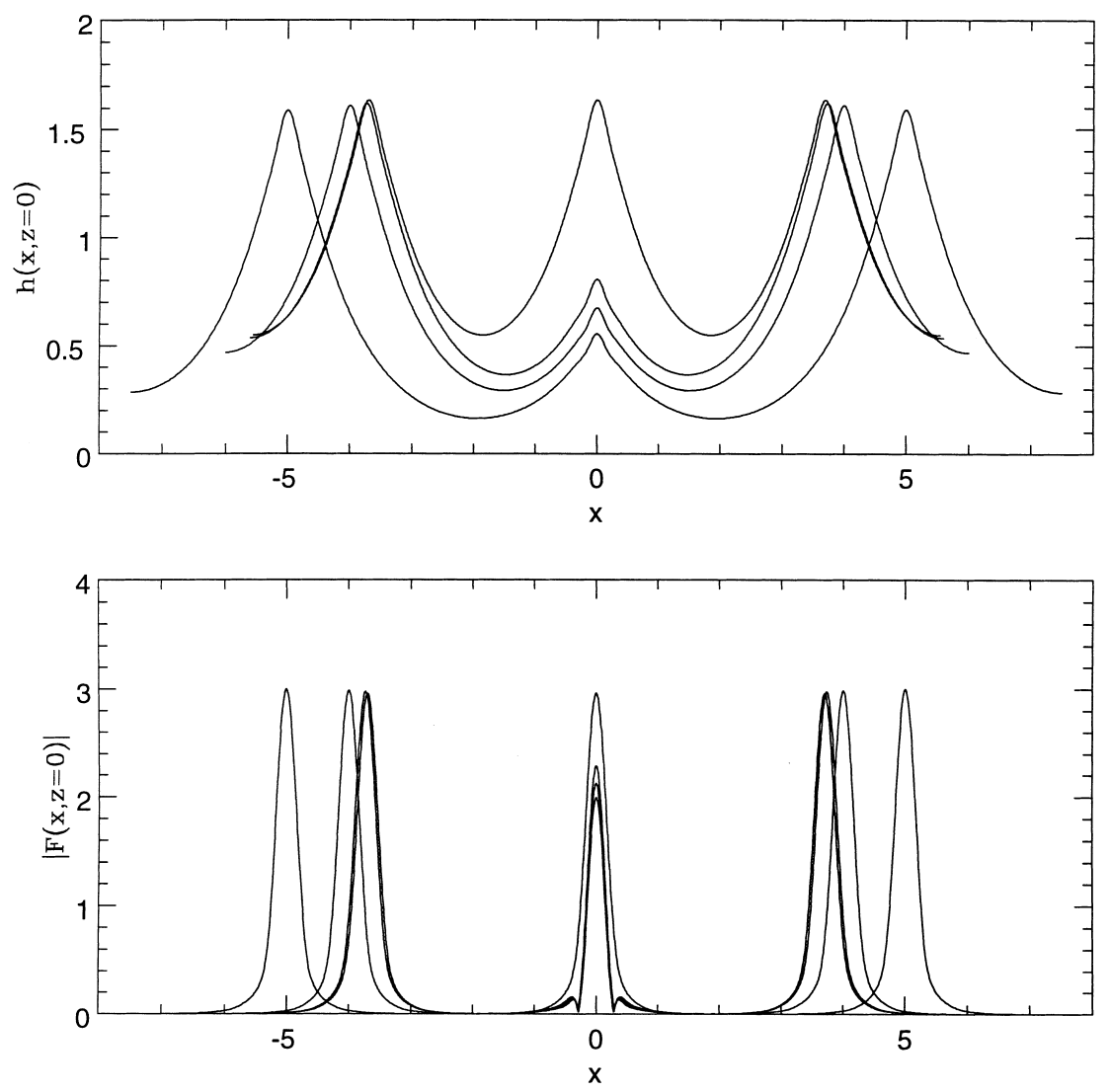

Fig. 25. The bifurcation of the pattern "101" of a three pixel array with self-defocusing nonlinearity. Curves are for interpixel spacing of $S=5,4,3.733$ and 3.7, respectively. $\Gamma=-0.005$ in all curves.

In both Figs. 25 and 26, there is a clear separation between the distributions of the electric fields. Even when switching starts, the separation is still well defined. This indicates that diffusion accounts for almost all the crosstalk. Diffraction participates only by affecting the operation gap and the shape of each pixel.

If diffraction does not play any significant role in crosstalk, then an overall phase difference between the input fields of one pixel and that of the other pixels should not have any effect on the operation of an array. Such considerations may be important, for example, for beam multiplexing. It will not change the solution locally. But it can affect the interaction between the pixel and its neighbors. In fact, phase encoding has been used as a technique to wipe out memories of diffraction only all-optical arrays [12,15].

Here, because diffractive crosstalk only occurs at the exponential tail of the input pulses, its effect is very weak. We have observed that if the phase of the "off" pixel is shifted by $180^{\circ}$, the optimal separation of the array barely changes.

\subsection{Estimating packing density for narrow input}

For narrow inputs, the theory of Section 5 can be applied to optical arrays. In this case, the model is reduced to (5.5) which is equivalent to a two-dimensional map:

$$
A_{n+1}=2 c A_{n}-s\left(1+r_{\mathrm{b}}^{2}\right) f_{\mathcal{F}}\left(\delta+2 A_{n}\right) P_{0}-B_{n}, \quad B_{n+1}=A_{n} .
$$



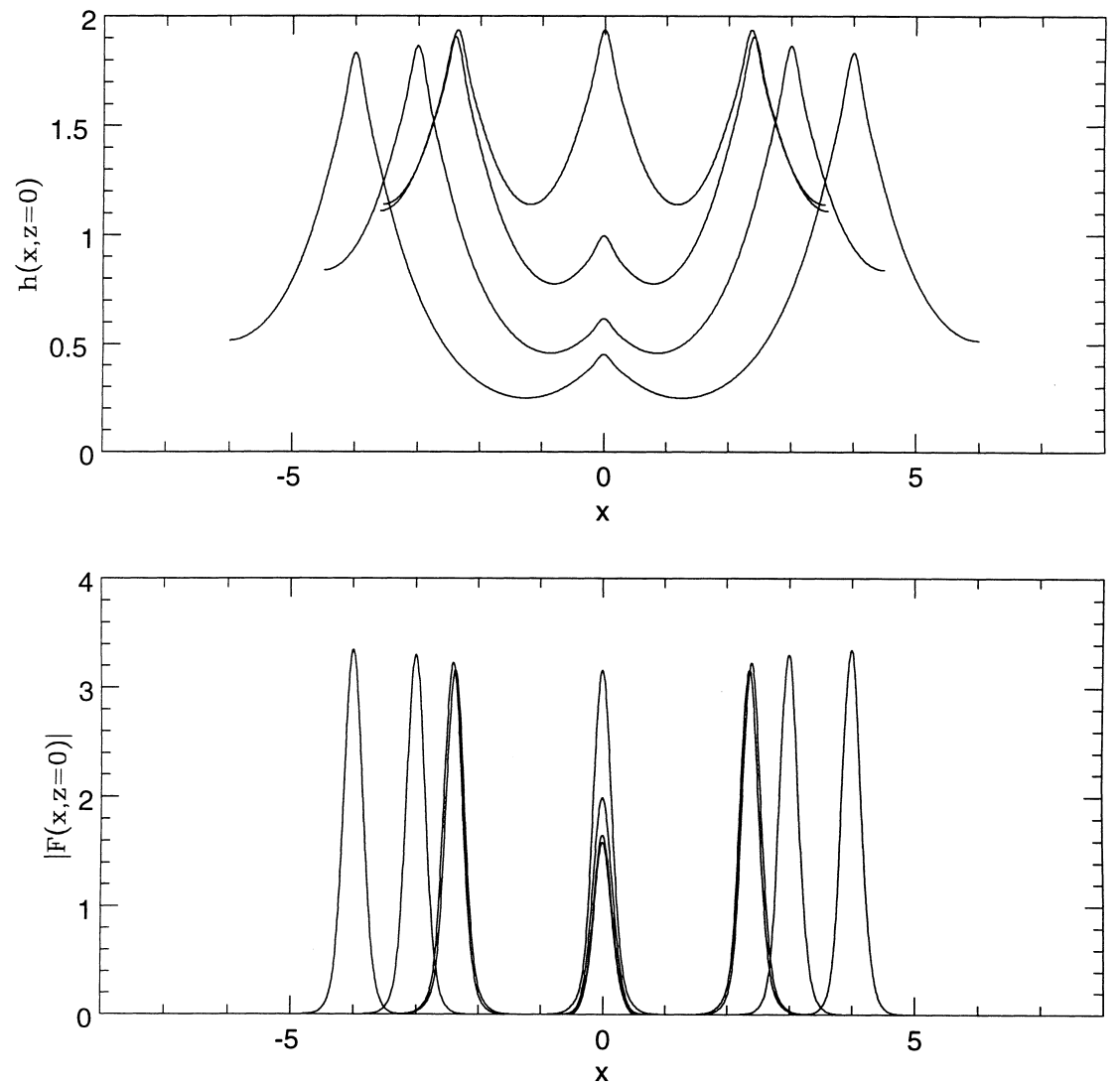

Fig. 26. The bifurcation of the pattern "101" of a three pixel array with self-focusing nonlinearity. Curves are for interpixel spacing of $S=4,3,2.4$ and 2.367 , respectively. $\Gamma=0.005$ in all curves.

Once $f_{\mathcal{F}}(\varphi)$ is computed, the minimal packing density can be estimated in two ways [4,5]: (i) numerically finding a Smale horseshoe, or (ii) graphically solving the optimal packing density of a three pixel array. Estimating the optimal packing of a three pixel array is easier than numerically finding a Smale horseshoe, and provides not only a lower bound of the optimal packing density of an infinite array, but also a good estimate of it [2].

The estimation for Fig. 26 yields $S_{\mathrm{c}}=2.5427$. That agrees well with the numerical result. As discussed in Section 5.4 , the use of the theory is not justifiable for input width $w=0.2$. Still, the reduced map works reasonably well in estimating optimal memory densities.

In the case of self-defocusing nonlinearity, the reduced model does not work well even for a single pixel bistability when $w=0.2$. Bistability ceases to exist when $\Gamma=-0.005(\mathcal{F}=0.25)$. Hence the theory will not be able to fit Fig. 25. For this reason, an input with $w=0.05$ and $\Gamma=0.0002$ is used in the numerical computation. These numbers correspond to $\mathcal{F}=0.08$. The theoretical result is $S_{\mathrm{c}}=3.6650$. The numerical estimation, carried out on a Cray C-90, is roughly 3.8333 . Again the theory is proved to be qualitatively accurate.

\subsection{Switching}

An optical array should not only be able to store information, but it also must be addressable. Addressing is usually achieved by some addressing signals, which are typically short-lived square pulses, i.e., pulses which lasts for only a short period of time. 


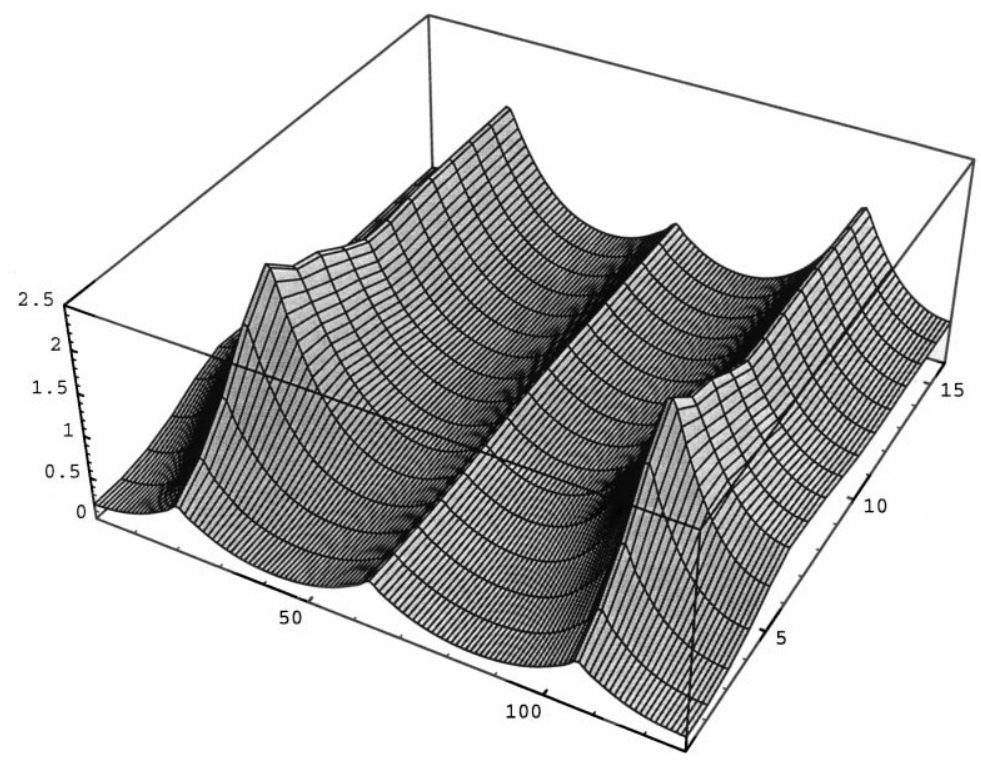

Fig. 27. Addressing the pattern "101" of a three pixel array when $S$ is near its critical value. $S=3.733$ and $\Gamma=-0.005$.

Fig. 27 shows that we can successfully address pattern "101" in a (defocuing) three pixel array, even when the pixel separation is near its critical value. The addressing beam has power $P_{\text {addr }}=1.0$ and lasts for a duration of 10 transits. To ensure a smooth addressing, the addressing beam is ramped up and down over a time of two transits. We will count half of the turning "on" and "off" times towards the addressing time. So the total addressing time is 12 transit times.

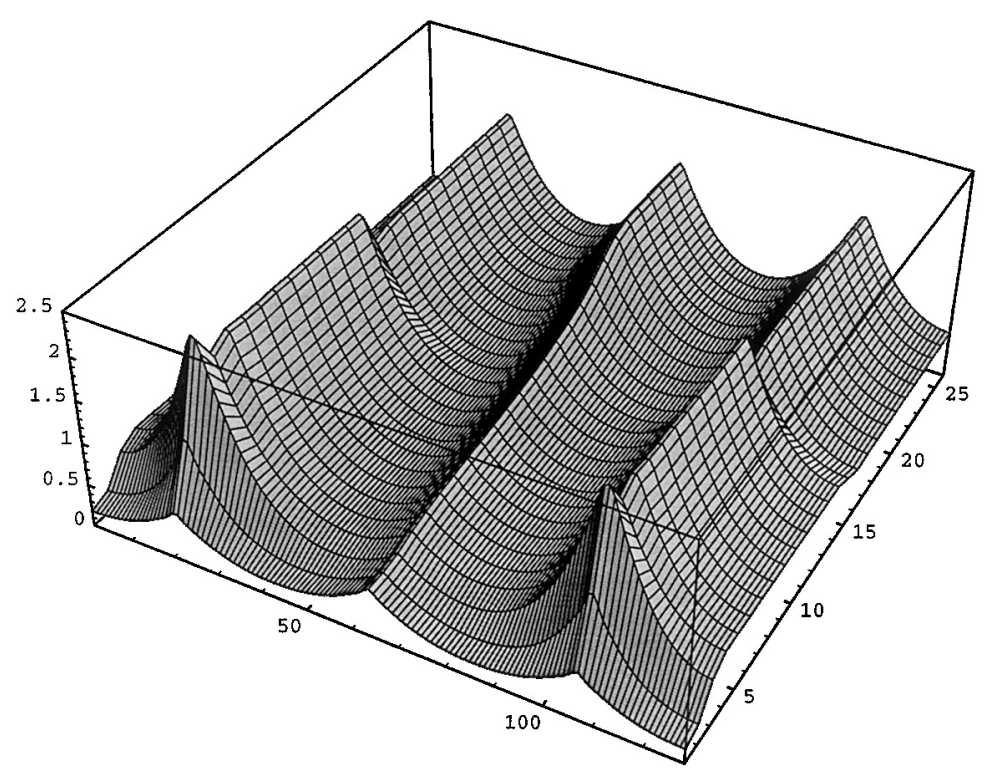

Fig. 28. A prolonged addressing that turns on the central bit of the pattern "101". Parameters are the same as Fig. 27 except that the addressing lasts for 22 transits. 

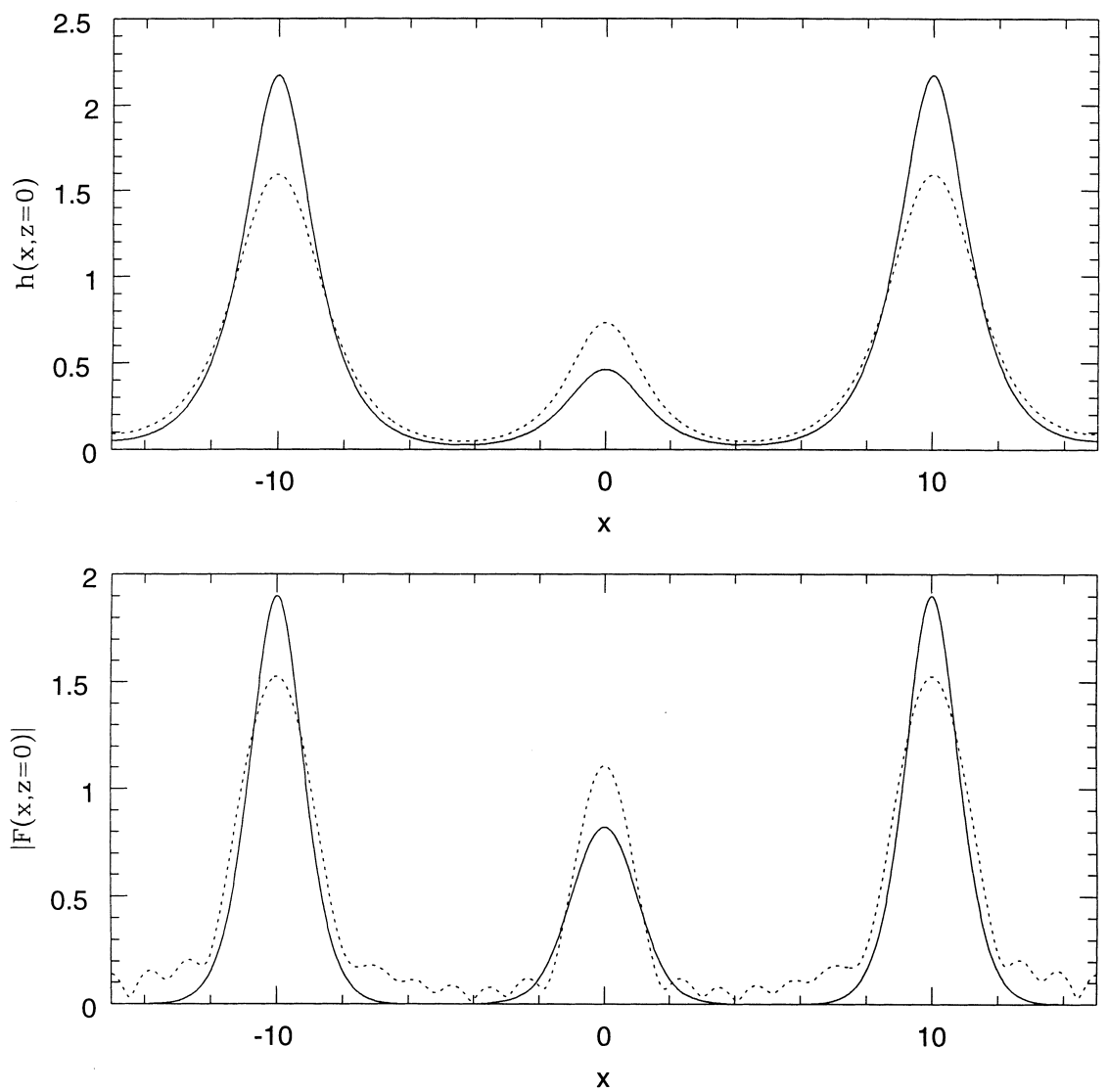

Fig. 29. Solutions of three pixel arrays with a wide input. $\Gamma=0.2$ for the solid lines and -0.2 for the dotted lines. $S=10, w=1.414$ in both cases.

In this case, the switching waves observed in [18] are not seen. Our explanation is that the switching by addressing signals is much faster than the switching due to crosstalk. If the addressing time is very short, the induced switching may not have time to respond. If the addressing lasts longer, the "off" bit will be eventually switched by crosstalk (see Fig. 28). For this reason, a short strong addressing signal is preferred to a weak but long one. This observation is similar to one made for diffusive optical arrays [18].

It should be noted that for fixed switching power, there is a lower limit on the switch duration. It is determined by a pulse area criterion where the product of power and duration is a constant $[18,20]$. This criterion works for very strong switching signals.

\subsection{Diffraction effects on arrays with wide input}

Section 4 has shown that the sign of nonlinearity becomes more important for diffractive effects on bistability when the input beam is wider. Fig. 29 depicts solutions with input width $w=1.414$ and $\Gamma= \pm 0.2$. The difference between the two signs of nonlinearity is clearly greater than the difference between Figs. 25 and 26, where the input width was $w=0.2$. Specifically, in the focusing case, the electric fields are well separated. While in the defocusing case, there is a clear interaction between the electric fields of the "on" bits and the "off" bit. In fact, this interaction seems to be even stronger than the diffusive interaction. 
This diffractive crosstalk will enhance diffusive crosstalk and speed up switching. The optimal separation is found to be $S_{\mathrm{c}}=7.5$ in the self-defocusing case and 3.733 in the self-focusing case. Again, focusing nonlinearity is preferred to defocusing nonlinearity for optical arrays.

\section{Conclusion}

Optical bistability and optical arrays in the presence of both diffraction and diffusion have been studied analytically and numerically. Bistability is very different for different types of nonlinearity. Diffraction tends to open up the gap between the two states when the nonlinearity is focusing, and close the gap when nonlinearity is defocusing. The smaller operation gap forces coarser pixel packing in a defocusing optical array. This happens even though diffractive crosstalk is not significant.

Nonlinear behavior typically observed in nonlinear Schrödinger equations is not seen in our full model because diffusion smoothes the refractive index and hence weakens nonlinearity. This observation leads to the reduction of the full model to a "reduced map". The reduction builds diffraction into a nonlinear response function $f_{\mathcal{F}}(\varphi)$. The result is a renormalization of Firth's map for the purely diffusive case. Using this reduced map, diffractive effects on bistability are explained qualitatively and optimal packing densities of optical arrays are estimated numerically.

\section{Appendix A. Numerical algorithm}

An important property of system (1.1)-(1.3) is that each of the three equations has its own characteristic direction. We define

$$
\xi=\frac{1}{2}(t+z), \quad \eta=\frac{1}{2}(t-z)
$$

and rewrite (1.1)-(1.3) as

$$
\begin{aligned}
& \frac{\partial F(\xi, \eta, x)}{\partial \xi}-\mathrm{i} \Gamma \frac{\partial^{2}}{\partial x^{2}} F(\xi, \eta, x)=\mathrm{i} h F(\xi, \eta, x), \quad \frac{\partial B(\xi, \eta, x)}{\partial \eta}-\mathrm{i} \Gamma \frac{\partial^{2}}{\partial x^{2}} B(\xi, \eta, x)=\mathrm{i} h B(\xi, \eta, x), \\
& \tau \frac{\partial h(t, z, x)}{\partial t}=\frac{\partial^{2}}{\partial x^{2}} h(t, z, x)-h(t, z, x)+n_{2}\left(|F(t, z, x)|^{2}+|B(t, z, x)|^{2}\right) .
\end{aligned}
$$

Notice that we have retained the characteristic directions of each equation as its own coordinates. A split-step method is then applied on these new coordinates. The details of the algorithm is as follows:

- The linear step

- Fast Fourier transform

$$
\begin{aligned}
& \tilde{F}\left(t_{i}, z_{j}, \omega_{l}\right)=\sum_{k=1}^{M} \mathrm{e}^{\mathrm{i} \omega_{l} x_{k}} F\left(t_{i}, z_{j}, x_{k}\right), \quad \tilde{B}\left(t_{i}, z_{j}, \omega_{l}\right)=\sum_{k=1}^{M} \mathrm{e}^{\mathrm{i} \omega_{l} x_{k}} B\left(t_{i}, z_{j}, x_{k}\right), \\
& \tilde{h}\left(t_{i}, z_{j}, \omega_{l}\right)=\sum_{k=1}^{M} \mathrm{e}^{\mathrm{i} \omega_{l} x_{k}} h\left(t_{i}, z_{j}, x_{k}\right) .
\end{aligned}
$$

- Solution in the spectral space

$$
\begin{aligned}
& \tilde{F}\left(t_{i}+\Delta, z_{j}, \omega_{l}\right)=\tilde{F}\left(t_{i}, z_{j}, \omega_{l}\right) \mathrm{e}^{-\mathrm{i} \Gamma \omega_{l}^{2} \Delta}, \quad \tilde{B}\left(t_{i}+\Delta, z_{j}, \omega_{l}\right)=, \tilde{B}\left(t_{i}, z_{j}, \omega_{l}\right) \mathrm{e}^{-\mathrm{i} \Gamma \omega_{l}^{2} \Delta} \\
& \tilde{h}\left(t_{i}+\Delta, z_{j}, \omega_{l}\right)=\tilde{h}\left(t_{i}, z_{j}, \omega_{l}\right) \mathrm{e}^{(-1 / \tau) \omega_{l}^{2} \Delta} .
\end{aligned}
$$


- Backward fast Fourier transform

$$
\begin{aligned}
& F\left(t_{i}+\Delta, z_{j}, x_{k}\right)=\frac{1}{2 \pi} \sum_{l=1}^{M} \mathrm{e}^{-\mathrm{i} \omega_{l} x_{k}} \tilde{F}\left(t_{i}+\Delta, z_{j}, \omega_{l}\right), \\
& B\left(t_{i}+\Delta, z_{j}, x_{k}\right)=\frac{1}{2 \pi} \sum_{l=1}^{M} \mathrm{e}^{-\mathrm{i} \omega_{l}, x_{k}} \tilde{B}\left(t_{i}+\Delta, z_{j}, \omega_{l}\right), \\
& h\left(t_{i}+\Delta, z_{j}, x_{k}\right)=\frac{1}{2 \pi} \sum_{l=1}^{M} \mathrm{e}^{-\mathrm{i} \omega_{l} x_{k}} \tilde{h}\left(t_{i}+\Delta, z_{j}, \omega_{l}\right) .
\end{aligned}
$$

- The nonlinear step

$$
\begin{aligned}
& F\left(t_{i}+\Delta, z_{j}, x_{k}\right)=F\left(t_{i}, z_{j}-\Delta, x_{k}\right) \mathrm{e}^{\mathrm{i}(\Delta / 2)\left(h\left(t_{i}, z_{j}-\Delta, x_{k}\right)+h\left(t_{i}+\Delta, z_{j}, x_{k}\right)\right)}, \\
& B\left(t_{i}+\Delta, z_{j}, x_{k}\right)=B\left(t_{i}, z_{j}+\Delta, x_{k}\right) \mathrm{e}^{\mathrm{i}(\Delta / 2)\left(h\left(t_{i}, z_{j}+\Delta, x_{k}\right)+h\left(t_{i}+\Delta, z_{j}, x_{k}\right)\right)}, \\
& \tau \frac{h\left(t_{i}+\Delta, z_{j}, x_{k}\right)-h\left(t_{i}, z_{j}, x_{k}\right)}{\Delta}=-\frac{\Delta}{2}\left(h\left(t_{i}, z_{j}, x_{k}\right)+h\left(t_{i}+\Delta, z_{j}, x_{k}\right)\right) \\
& \quad+n_{2} \frac{\Delta}{2}\left(\left|F\left(t_{i}, z_{j}, x_{k}\right)\right|^{2}+\left|B\left(t_{i}, z_{j}, x_{k}\right)\right|^{2}\right)+n_{2} \frac{\Delta}{2}\left(\left|F\left(t_{i}, z_{j}-\Delta, x_{k}\right)\right|^{2}+\left|B\left(t_{i}, z_{j}+\Delta, x_{k}\right)\right|^{2}\right) .
\end{aligned}
$$

\section{References}

[1] N.B. Abraham, W.J. Firth, Overview of transverse effects in nonlinear-optical systems, J. Opt. Soc. Am. B 7 (1990) 951-962.

[2] Y. Chen, Diffraction effects on diffusive optical bistability and optical memory, Ph.D. Dissertation, Princeton University, Princeton, NJs, 1998.

[3] Y. Chen, D.W. McLaughlin, Focusing-defocusing effects for diffusion dominated bistable optical arrays, J. Opt. Soc. Am. B 16 (1999) 1087-1098.

[4] W.J. Firth, Optically bistable arrays and chaotic dynamics, Phys. Lett. A 125 (1987) 375-379.

[5] W.J. Firth, Optical memory and spatial chaos, Phys. Rev. Lett. 61 (1988) 329-332.

[6] W.J. Firth, E. Abraham, E.M. Wright, I. Galbraith, B.S. Wherrett, Diffusion, diffraction and reflection in semiconductor OB devices, Philos. Trans. R. Soc. London A 313 (1984) 299-306.

[7] W.J. Firth, I. Galbraith, Diffusive transverse coupling of bistable elements — switching waves and crosstalk, J. Quant. Elect. QE-21 (1985) 1399-1403.

[8] W.J. Firth, I. Galbraith, E.M. Wright, Diffusion and diffraction in dispersive optical bistability, J. Opt. Soc. Am. B 2 (1985) 1005-1009.

[9] H.M. Gibbs, Optical Bistability: Controlling Light with Light, Academic Press, London, 1985.

[10] D. Gottlieb, S.A. Orszag, Numerical analysis of spectral methods: theory and application, Society for Industrial and Applied Mathematics, Philadelphia, 1977.

[11] D.J. Hagan, H.A. MacKenzie, H.A. Al-Atlar, W.J. Firth, Carrier diffusion measurements in InSb by the angular dependence of degenerate four-wave mixing, Opt. Lett. 10 (1985) 187-189.

[12] G.S. McDonald, Spatial solitary wave optical memory, Ph.D. Dissertation, Strathclyde University, Glasgow, Scotland, 1989.

[13] G.S. McDonald, W.J. Firth, All-optical switching in a nonlinear resonator, J. Mod. Opt. 37 (1990) 613-626.

[14] G.S. McDonald, W.J. Firth, Spatial solitary-wave optical memory, J. Opt. Soc. Am. B 7 (1990) 1328-1335.

[15] G.S. McDonald, W.J. Firth, Switching dynamics of spatial solitary wave pixels, J. Opt. Soc. Am. B 10 (1993) 1081-1089.

[16] J.V. Moloney, M. Sargent III, H.M. Gibbs, Transverse effects in absorptive optical bistability, Opt. Comm. 44 (1983) $289-292$.

[17] M.I. Nathan, J.C. Marinace, R.F. Rutz, A.E. Michel, G.J. Lasher, GaAs injection laser with novel mode control and switching properties, J. Appl. Phys. 36 (1965) 473-480.

[18] K.D. Stephen, W.J. Firth, Absorptive optically bistable arrays, J. Mod. Opt. 37 (1990) 627-638.

[19] A. Szoke, V. Daneu, J. Goldnar, N.A. Kurnit, Bistable optical element and its applications, Appl. Phys. Lett. 15 (1969) $376-379$.

[20] D. Weaire, C. O'Carroll, C. Wickham, Dispersive optical bistability with diffusion: a scaling law, Europhys. Lett. 8 (1989) 25-28. 